Check for updates

Cite this: RSC Adv., 2020, 10, 11694

Received 22nd January 2020

Accepted 26th February 2020

DOI: 10.1039/d0ra00681e

rsc.li/rsc-advances

\section{Design, synthesis and biological evaluation of novel amide-linked $18 \beta$-glycyrrhetinic acid derivatives as novel ALK inhibitors $\dagger$}

\begin{abstract}
Dong Cai, (D) ${ }^{a}$ Zhi hua Zhang, ${ }^{b}$ Yu Chen, ${ }^{c}$ Chao Ruan, ${ }^{d}$ Sheng qiang Li, ${ }^{d}$ Shi qin Chen ${ }^{d}$ and Lian shan Chen ${ }^{\star d}$

A series of novel amide-linked $18 \beta$-glycyrrhetinic acid derivatives were developed by incorporating substituted piperazine amide fragments into the $\mathrm{C} 30-\mathrm{COOH}$ of $18 \beta$-glycyrrhetinic acid scaffold. The synthesized compounds were evaluated for their anticancer activity against Karpas299, A549, HepG2, MCF-7, and PC-3 cell lines by MTT assay. Besides, some compounds with electron-withdrawing groups on phenyl moieties exhibited noticeable antiproliferative activity. The most potent compound $4 a$ was also found to be non-toxic to normal human hepatocytes LO2 cells. The compound 4a exhibited moderate inhibitory activity against wild-type ALK with an $I_{50}$ value of $203.56 \mathrm{nM}$ and relatively weak potent activity to $\mathrm{c}-$ Met $\left(\mathrm{IC}_{50}>1000 \mathrm{nM}\right)$. Molecular docking studies were performed to explore the diversification in bonding patterns between the compound $4 a$ and Crizotinib.
\end{abstract}

\section{Introduction}

Nowadays, most tyrosine kinase inhibitors bind in a nearly identical position to that of the ATP in kinases by forming hydrogen bonds to hinge residues and by hydrophobic interactions in and around the region taken up by the adenine ring of ATP. Several tyrosine kinase inhibitors can form additional hydrogen bond interactions and hydrophobic interactions with the DFG residues of the activation-loop adjacent to the region taken up by ATP. Those tyrosine kinase inhibitors are termed as typical type I and II inhibitors, respectively. ${ }^{\mathbf{1 , 2}}$

A general pharmacophore model of type II inhibitors covers three regions, namely, hinge-region binding, linker and tail moieties. In particular, a hydrogen bond donor-acceptor pair and a hydrophobic fragment in the tail moiety are capable of selectively taking up allosteric pocket, exhibiting an advantage over type I inhibitors. ${ }^{2,3}$

18 $\beta$-Glycyrrhetinic acid (18 $\beta$-GA, Fig. 1$)$ is an inexpensive and available triterpene extracted from the roots of licorice plants (Glycyrrhiza glabra); its derivatives exhibit remarkable cytotoxic and pharmacological activities, in particular antitumor

${ }^{a}$ College of Public Basic Sciences, Jinzhou Medical University, Jinzhou, 121001, China ${ }^{b}$ School of Chemical and Environmental Engineering, Liaoning University of Technology, Jinzhou, 121001, China

${ }^{c}$ School of Life Science and Biopharmaceutics, Shenyang Pharmaceutical University, Shenyang, 110016, China

${ }^{d}$ College of Pharmacy, Jinzhou Medical University, Jinzhou, 121001, China. E-mail: 509205162@QQ.com

$\dagger$ Electronic supplementary information (ESI) available. See DOI: 10.1039/d0ra00681e activity. ${ }^{4-6}$ The $18 \beta$-GA nucleus, a feasible structure for in-depth pharmaceutical exploration and for development of new potential antiproliferative drug candidates, has aroused extensive attention from medicinal chemists over the last decade..$^{7-11}$ The presence of polar functional groups (C3-OH and C30$\mathrm{COOH})$ in its structure could impact the biological activities of the mentioned analogues. ${ }^{12}$

Inspired by the mentioned developments and facts, our previous work was continued in structural modification of $18 \beta$ GA. ${ }^{7}$ In the present study, substituted piperazine amide fragments were introduced into the $\mathrm{C} 30-\mathrm{COOH}$ of $18 \beta-\mathrm{GA}$, and a novel series of amide-linked derivatives was designed as potential antitumor inhibitors. To assess the effect of different substituents on the piperazine amide fragment, the substitution of phenyl or benzyl group was modified, and the aromatic ring was altered (Fig. 2).

Piperidine carboxamide A (Fig. 3a), an anaplastic lymphoma kinase (ALK) inhibitor, exhibits excellent cytotoxic activity

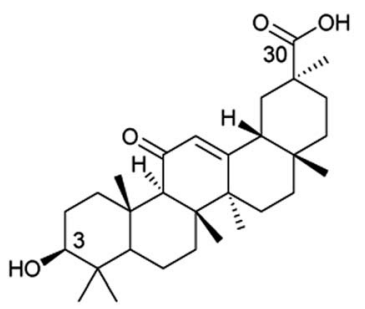

$18 \beta$-glycyrrhetinic acid

Fig. 1 Structure of $18 \beta-G A$. 


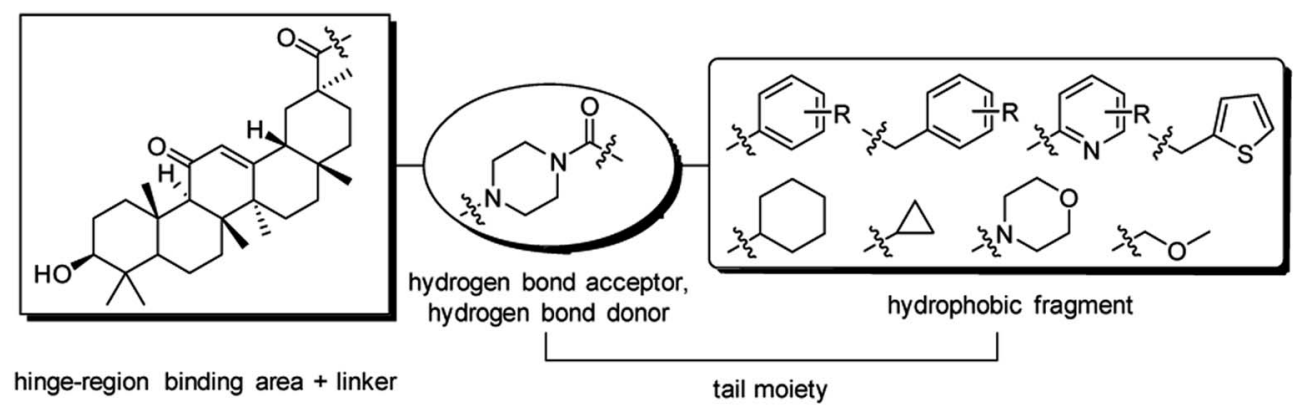

Fig. 2 Rational design of the target compounds.

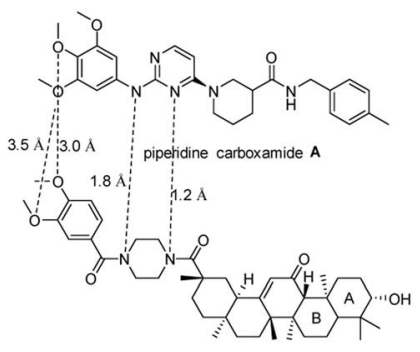

a)

b)

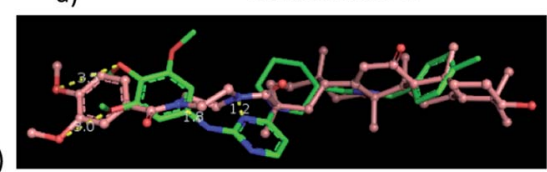

Fig. 3 (a) Piperidine carboxamide $A$ and compound $4 a$, (b) overlay of piperidine carboxamide $A$ (stick; from cocrystal structure, PDB code: $4 \mathrm{DCE}$ ) and the energy minimized structure for compound $4 \mathrm{a}$ (ball and stick).

against ALK-positive Karpas-299 cells $\left(\mathrm{IC}_{50}=0.384 \mu \mathrm{M}\right)$. Moreover, such bioactive compound displayed significant activity in the ALK enzyme assay $\left(\mathrm{IC}_{50}=0.174 \mu \mathrm{M}\right)$, probably causing a reduction in the phosphorylation levels of ALK downstream effectors. $^{13}$

Chemical compounds with nearly identical threedimensional (3D) structures are likely to exhibit similar activities. The similarity can be computed based upon steric, electronic, and/or other physical properties. ${ }^{14}$ Molecular overlay provided insights into possible improvements in potency and selectivity of the designed compounds. In the present study, the energy minimization conformation of the target compound $\mathbf{4 a}$ was aligned to the crystal conformation of piperidine carboxamide A using the molecular overlay option of Discovery Studio 3.5 suite. Fig. $3 \mathrm{~b}$ suggests that the distance of methoxy groups on the benzyl fragments of two molecules reached 3.0 and $3.5 \AA$, respectively. Given the instability of linear molecular conformations, it is a relatively acceptable superposition between the mentioned two the lipophilic methoxyphenyl fragments. Moreover, the two nitrogen atom on piperazine ring are very close to the corresponding nitrogen atom on the 2-aminopyrimidine ring. Another overlapping feature was the presence of the alicyclic fragment of A and B rings of the target compound $\mathbf{4 a}$, as well as the $p$-methyl benzyl group from piperidine carboxamide A. The 3D molecular similarity score was 0.3049 . The maximum shape similarity of molecules is expressed by score 1 and the minimum similarity is denoted by score $0 .^{15-17}$

According to the mentioned results, the novel amide-linked 18 $\beta$-GA derivatives might exhibit potential antiproliferative activity.

\section{Results and discussion}

\subsection{Chemistry}

The synthesis of amide-linked $18 \beta-G A$ derivatives $(\mathbf{4 a - 4 v})$ was depicted in Scheme 1. To produce compound (2), 18 $\beta$-GA was reacted with 1-Boc-piperazine in the presence of ethyldimethylaminopropyl-carbodiimide hydrochloride (EDCl), 1hydroxybenzotriazole (HOBt) and triethylamine. Subsequently, the compound (2) was stirred in the solution of trifluoroacetate (TFA) and dichloromethane $(1: 1)$ to remove Boc group. Without being further purified, monoamide (3) was acylated with acid chlorides in the presence of triethylamine to produce the target compounds (4a-4v).

A simple and efficient approach for amide bond formation is based on the reaction of substituted carboxylic acid and amine in the presence of coupling reagents. ${ }^{18-20}$ Monoamide (3) can also be generated by treating of compound (2) with piperazine in the presence of EDCl, HOBt, and triethylamine. However, the corresponding monoamide (3) could be obtained in relatively low isolated yields, the reaction was complicated by the competing diamidation to form symmetric bisamide (5). As shown in Scheme 2, the amount of the symmetric bisamide (5) generated was determined by the reaction conditions. For instance, no bisamide (5) was identified by treating the compound (2) with piperazine (1.0 equiv.) in the presence of EDCl, HOBt, and triethylamine in $\mathrm{CH}_{3} \mathrm{CN}$ at room temperature for $24 \mathrm{~h}$. However, an intermediate (6) was largely isolated from the reaction mixture. ${ }^{7}$ The intermediate (6) did not react with piperazine at low reaction temperatures even after the extended reaction time (up to $48 \mathrm{~h}$ ). Nevertheless, the above reaction was performed in refluxing $\mathrm{CH}_{3} \mathrm{CN}$, and intermediate (6) could be fully converted into bisamide (5) and trace amount of the desired monoamide (3).

The steric hindrance around the C-30 ester group of intermediate (6) significantly impacted the nucleophilic 


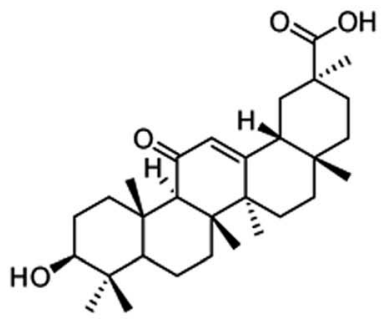

1<smiles>CC(C)(C)OC(=O)N1CCN(C(=O)[C@]2(C)CC[C@]3(C)C4=CC(=O)C3[C@@]3(C)CCC5C(C)(C)[C@H](O)CC[C@]5(C)[C@]3(C)[C@@H]4C2)CC1</smiles>

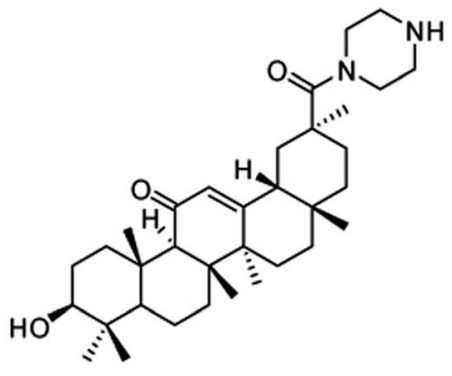

3

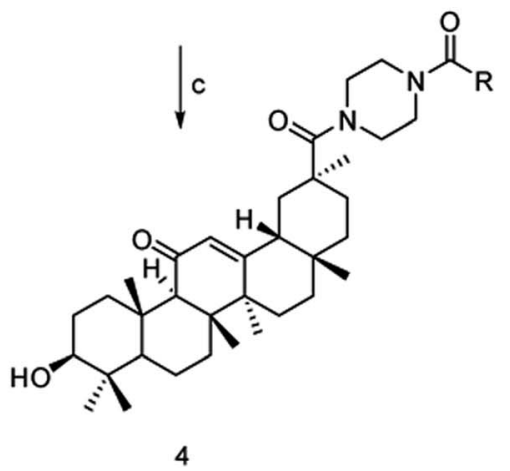

Scheme 1 Synthesis of compound 4. Reagents and conditions: (a) 1-Boc-piperazine, $\mathrm{CH}_{3} \mathrm{CN}, \mathrm{NEt}$, $\mathrm{EDCl}, \mathrm{HOBt}, \mathrm{reflux}, 24 \mathrm{~h}$; (b) TFA, CH${ }_{2} \mathrm{Cl}_{2}, \mathrm{O} /$ $25{ }^{\circ} \mathrm{C}$; (c) substituted acyl chloride, $\mathrm{CH}_{2} \mathrm{Cl}_{2}, \mathrm{Et}_{3} \mathrm{~N}$, r.t.

substitution. The lowest-energy conformer of intermediate (6) and piperazine was achieved by MM2 calculations in ChemBio 3D Ultra 12.0. As shown in Fig. 4, the piperazine was difficult to 4c $\mathrm{R}=3,5-\mathrm{diCH}_{3}$ phenyl, 4f $\mathrm{R}=2,4-\mathrm{diCl}$ phenyl, 4j $\mathrm{R}=3-\mathrm{Br}$ phenyl, $4 \mathrm{~m} \mathrm{R}=2,4-\mathrm{diF}$ phenyl, $4 \mathrm{p} \mathrm{R}=4-\mathrm{CF}_{3}$ phenyl, 4s R=Cyclopropyl, 4v R=4-F benzyl, 4y $\mathrm{R}=\mathrm{CH}_{3} \mathrm{OCH}_{2}$, get close to C-30 because of two bulky groups $(1 \mathrm{H}$-benzo $[d]$ $[1,2,3]$ triazol-1-yl group and scaffold of $18 \beta-\mathrm{GA})$.

Next, the effect of feeding sequence of above reaction was investigated. To a solution of piperazine ( 4.0 equiv.) in refluxing

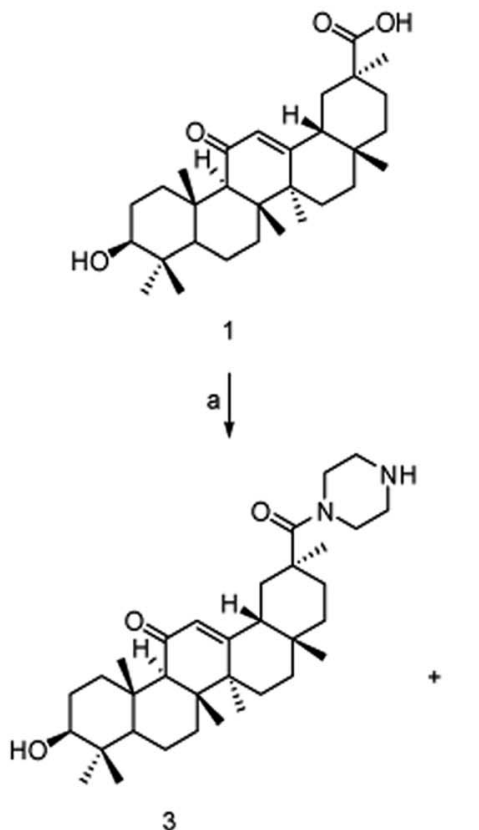<smiles>C1CCCCC1</smiles><smiles>c1ccc2[nH]nnc2c1</smiles>

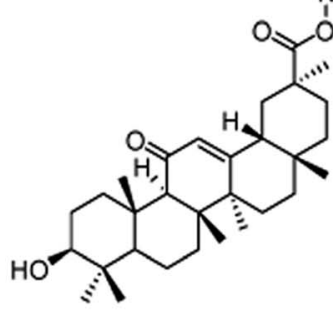

6<smiles>[C]1[C+]=CC=C1</smiles>

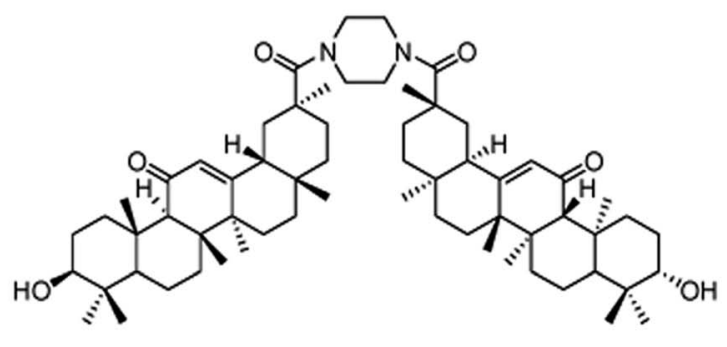

5

Scheme 2 Synthesis of compound 3, 5, and 6. Reagents and conditions: (a) piperazine, $\mathrm{CH}_{3} \mathrm{CN}, \mathrm{NEt}_{3}, \mathrm{EDCl}, \mathrm{HOBt}$, reflux, $12 \mathrm{~h}$; (b) piperazine, $\mathrm{CH}_{3} \mathrm{CN}, \mathrm{NEt}_{3}, \mathrm{EDCl}, \mathrm{HOBt}$, r.t.; (c) piperazine, $\mathrm{CH}_{3} \mathrm{CN}$, reflux. 


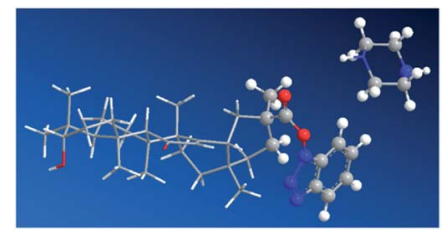

Fig. 4 The energy minimized intermediate (6) and piperazine.

$\mathrm{CH}_{3} \mathrm{CN}$, intermediate (6) solution (1.0 equiv.) was added dropwise and then stirred for $12 \mathrm{~h}$. As shown in Scheme 2, under the reaction, bisamide (5) and the desired monoamide (3) were formed, and the isolated yields reached $53.7 \%$ and $40.8 \%$, respectively.

Condensation of nearly equimolecular amounts of monoamide (3) with substituted acyl chloride in $\mathrm{CH}_{2} \mathrm{Cl}_{2}$ with triethylamine at room temperature affords target compounds (4a4v) with $84.8-94.6 \%$ yields. The $\mathrm{C} 3-\mathrm{OH}$ of $18 \beta-\mathrm{GA}$ did not interfere with these amidation reactions. However, the amidation of monoamide (3) with 2-(4-chlorophenyl)acetyl chloride was carried out in the solution of triethylamine and $\mathrm{CH}_{2} \mathrm{Cl}_{2}$ at $40{ }^{\circ} \mathrm{C}$, and competitive esterification to form the corresponding compound (7) in $38.4 \%$ isolated yield contributed to a lower yield of the target compound (4w). Moreover, if the acid chloride is excessively large and the reaction time is too long at room temperature, it will also lead to competitive esterification (Scheme 3).

\subsection{In vitro cell growth inhibitory activity}

To test the anticancer activity of synthesized compounds, antiproliferative activity of target compounds (4a-4v, 7) against HepG2 and Karpas299 was assessed by MTT assay. 18 $\beta$-GA and Crizotinib were used as a positive control. The results are expressed as the growth inhibition, as listed in Table 1. Most compounds exhibited prominent antiproliferative activities at a dose $\left(20 \mu \mathrm{g} \mathrm{mL} \mathrm{m}^{-1}\right)$, this was primarily attributed to the fact that the target compounds have a large molecular weight. All the investigated compounds were less activity than Crizotinib.

Preliminary SAR analyses suggested that the substituent properties and positions of the phenyl ring fragment were critical to modulate their antiproliferative activity. Table 1
Table 1 Antiproliferative activity of the target compounds (growth inhibition, \%)

\begin{tabular}{|c|c|c|c|c|}
\hline \multirow[b]{2}{*}{ Compound } & \multicolumn{2}{|l|}{ HepG2 } & \multicolumn{2}{|l|}{ Karpas299 } \\
\hline & $2 \mu \mathrm{g} \mathrm{mL}^{-1}$ & $20 \mu \mathrm{g} \mathrm{mL}^{-1}$ & $2 \mu \mathrm{g} \mathrm{mL}^{-1}$ & $20 \mu \mathrm{g} \mathrm{mL}^{-1}$ \\
\hline $4 a$ & 0.00 & 93.11 & 0.00 & 96.62 \\
\hline $4 \mathrm{~b}$ & 0.87 & 50.81 & 0.49 & 58.13 \\
\hline $4 c$ & 0.00 & 63.23 & 0.00 & 84.32 \\
\hline 4d & 0.00 & 68.04 & 0.00 & 91.46 \\
\hline $4 e$ & 0.00 & 11.96 & 0.00 & 18.28 \\
\hline 4f & 0.06 & 29.40 & 0.00 & 12.24 \\
\hline $4 \mathrm{~g}$ & 0.00 & 14.20 & 0.00 & 8.00 \\
\hline $4 \mathrm{~h}$ & 0.00 & 24.00 & 0.00 & 17.60 \\
\hline $4 \mathbf{i}$ & 0.00 & 19.10 & 0.00 & 12.76 \\
\hline $4 j$ & 0.10 & 34.51 & 0.00 & 6.62 \\
\hline $4 \mathbf{k}$ & 0.00 & 25.53 & 0.00 & 4.00 \\
\hline 41 & 4.26 & 51.12 & 0.00 & 69.32 \\
\hline $4 m$ & 0.00 & 40.24 & 0.0 & 53.31 \\
\hline $4 n$ & 0.00 & 32.73 & 0.00 & 26.27 \\
\hline 40 & 0.00 & 30.39 & 0.00 & 20.69 \\
\hline $4 p$ & 0.00 & 12.37 & 0.00 & 0.00 \\
\hline $4 q$ & 0.00 & 27.75 & 0.00 & 45.02 \\
\hline $4 r$ & 0.00 & 63.33 & 6.66 & 82.66 \\
\hline $4 s$ & 0.00 & 47.97 & 0.00 & 42.41 \\
\hline $4 t$ & 1.84 & 31.68 & 0.00 & 23.78 \\
\hline $4 u$ & 3.03 & 78.52 & 0.00 & 91.71 \\
\hline $4 v$ & 2.75 & 45.53 & 0.00 & 27.99 \\
\hline $4 w$ & 1.35 & 19.32 & 0.00 & 11.46 \\
\hline $4 x$ & 0.17 & 15.48 & 0.00 & 17.25 \\
\hline $4 y$ & 1.48 & 6.36 & 0.00 & 0.00 \\
\hline 7 & 0.00 & 0.00 & 0.00 & 0.00 \\
\hline $18 \beta-G A$ & 0.00 & 10.74 & 0.00 & 15.28 \\
\hline Crizotinib & 27.15 & 97.76 & 75.71 & 96.89 \\
\hline
\end{tabular}

presents that electron-withdrawing substituents on the phenyl ring displayed relatively poor antiproliferative activities against HepG2 and Karpas299 cells. Only the 3-fluorophenyl substituted derivative (41) showed over 50\% growth inhibitory activity at $20 \mu \mathrm{g} \mathrm{mL} \mathrm{mL}^{-1}$ against two test cells as compared with the target compounds (4e, $4 \mathbf{f}, \mathbf{4 g}, 4 \mathbf{h}, 4 \mathbf{i}, 4 \mathbf{j}, 4 \mathbf{k}, 4 \mathrm{~m}, \mathbf{4 n}, \mathbf{4 o}, \mathbf{4 p}$, $\mathbf{4 q )}$. The introduction of 6-chloronicotinoyl group at the identical position (4t) also significantly reduced activities against all of the tested cancer cell lines.

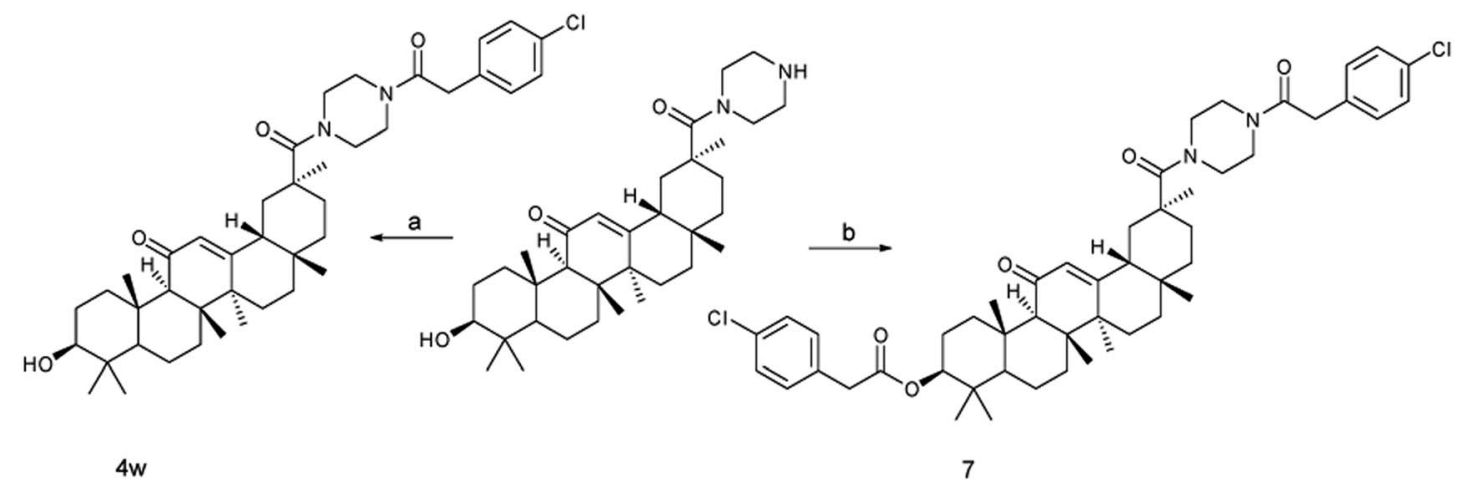

Scheme 3 Synthesis of compound $4 \mathrm{w}$, and 7. Reagents and conditions: (a) p-chlorophenylacetyl chloride, $\mathrm{CH}_{2} \mathrm{Cl}_{2}, \mathrm{Et}_{3} \mathrm{~N}, \mathrm{r.t}$; ; (b) $p$-chlorophenylacetyl chloride, $\mathrm{CH}_{2} \mathrm{Cl}_{2}, \mathrm{Et}_{3} \mathrm{~N}, 40{ }^{\circ} \mathrm{C}$. 
In contrast to the compounds with electron-withdrawing groups on the aromatic ring fragment, it was more favorable when the electron-donating group was added to the phenyl ring fragment (e.g. $\mathrm{CH}_{3} \mathrm{O}, \mathrm{CH}_{3}$ ). Compound (4a) exhibited nearly the identical cell growth inhibitory activity to Crizotinib against HepG2 and Karpas299 cells at the identical concentration of 20 $\mu \mathrm{g} \mathrm{mL}^{-1}$. 3-Methylphenyl substituted derivative (4d) also displayed good activity, especially for Karpas299 cell. Note that 3,5dimethoxyphenyl substituted derivative (4b) and 3,5-dimethylphenyl substituted derivative (4c) have drastically reduced inhibitory activity against HepG2 and Karpas299 cells, revealing that the substitution of the 3,5 position on the phenyl ring is not recommended.

When $\mathrm{R}$ is a substituted benzyl group, the compound (4v) containing 4-fluorobenzyl fragment exhibited better antiproliferative activity than that of compound ( $4 \mathbf{w}$ ) containing 4chlorobenzyl fragment. However, on the whole, the antitumor inhibition rate of these two compounds was below $50 \%$ at $20 \mu \mathrm{g}$ $\cdot \mathrm{mL}^{-1}$. Nevertheless, compound (4u) with thiophen-2methylene fragment displayed an outstanding potency against HepG2 and Karpas299 cells.

It is noteworthy that the target compounds (4r, 4s) with naphthenic substituted amides as the side chain showed an evident inhibitory effect against HepG2 and Karpas299 cells. In particular, $\mathrm{R}$ is cyclohexyl group, compounds (4r) exhibited notable cell growth inhibitory activity against Karpas299 cell (82.66\% at a concentration of $20 \mu \mathrm{g} \mathrm{mL} \mathrm{m}^{-1}$ ). Analogous compounds (4x, 4y) containing morpholine or methoxymethylene group, determined a significant decrease of efficacy. It is therefore indicated that the ether fragment could establish an unfavorable interaction with the receptor.

According to the structural features and corresponding antitumor activities of the compounds reported in the literature, ${ }^{\mathbf{1 0 , 2 1}}$ the antitumor activity of the selected compounds $\mathbf{4 a}$, 4c, 4d, 4r, and $4 \mathbf{u}$ was evaluated at the cellular level expressed by $\mathrm{IC}_{50}$ values against five cancer cell lines (Karpas299, A549, HepG2, MCF-7 and PC-3). Karpas299 is a typical anaplastic lymphoma kinase mutant-driven cancer cell line. Table 2 reveals that the most effective compound 4a exhibited superior antiproliferative effect only against Karpas299 and HepG2 cells with $\mathrm{IC}_{50}$ values of $6.51 \mu \mathrm{M}$, and $6.93 \mu \mathrm{M}$, respectively.

To determine whether the cell growth inhibitory effect of compound $\mathbf{4 a}$ is associated with a time- and concentrationdependent manner, the cells were treated by MTT cytotoxicity assay, and five concentration gradients of compound $4 \mathbf{a}$ were

Table 2 Antiproliferative activity $\left(\mathrm{IC}_{50}, \mu \mathrm{M}\right)$ of selected compound

\begin{tabular}{llllll}
\hline Compound & Karpas299 & A549 & HepG2 & MCF-7 & PC-3 \\
\hline 4a & 6.51 & $>40$ & 6.93 & 18.85 & 18.18 \\
$\mathbf{4 c}$ & 15.59 & $>40$ & 11.95 & $>40$ & 27.56 \\
$\mathbf{4 d}$ & 9.41 & $>40$ & 12.92 & $>40$ & 20.37 \\
$\mathbf{4 r}$ & 20.00 & 38.54 & 22.67 & 35.43 & 32.81 \\
$\mathbf{4 u}$ & 18.30 & $>40$ & 11.81 & $>40$ & 37.84 \\
18ß-GA & $>40$ & $>40$ & $>40$ & $>40$ & $>40$ \\
Crizotinib & 2.82 & 1.49 & 10.59 & 4.09 & 7.33
\end{tabular}

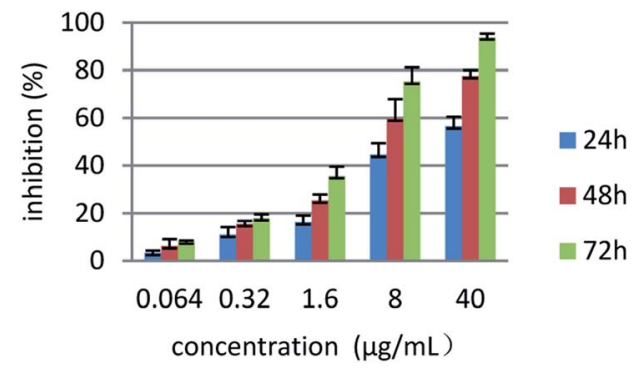

Fig. 5 The relationship between different concentrations and time of compound $4 \mathrm{a}$ and proliferation inhibitory effect. Data are means \pm SD of the inhibition (\%) from three independent experiments.

selected. After $24 \mathrm{~h}$ incubation, the HepG2 cells were treated for 24,48 and $72 \mathrm{~h}$ by compound $\mathbf{4 a}$ at the concentrations of 0.064 , $0.32,1.6,8.0$ and $40 \mu \mathrm{g} \bullet \mathrm{mL}^{-1}$. The result was shown in Fig. 5, compound $4 \mathbf{a}$ was observed in a significant time and concentration dependent manner to inhibit the proliferation of HepG2 cell.

According to the results, the compound 4a with the most potent antiproliferative activity was used for further processing. HepG2 cells and normal human hepatocytes LO2 cells were cultivated with compound $\mathbf{4 a}$ at increasing concentrations. A $48 \mathrm{~h}$ continuous drug exposure protocol was employed by the MTT assay. As shown in Fig. 6, compound 4a significantly inhibited the proliferation activity of HepG2 cells in a dosedependent manner. In contrast, compound $4 \mathbf{a}$ exhibited slight toxicity towards $\mathrm{LO} 2$ cells. As revealed from the results, the compound 4a might exhibit selective antiproliferative activity against human tumor cells.

\subsection{Kinase activities of compounds $4 a$ and $4 d$}

Given the mentioned results, the compounds $4 \mathbf{a}$ and $4 \mathbf{d}$ displayed potent, selective inhibitory activity against Karpas299 and HepG2 cells. The two compounds were taken for in-depth evaluation of the enzymatic inhibitory activity against wildtype ALK and c-MET/HGFR. Table 3 lists that the compounds 4a and 4d displayed moderate enzyme inhibitory activities against wild type $\mathrm{ALK}$ with $\mathrm{IC}_{50}$ values of $203.56 \mathrm{nM}$ and $686.19 \mathrm{nM}$, respectively, in comparison with that of the positive control Crizotinib $\left(\mathrm{IC}_{50}=11.21 \mathrm{nM}\right)$. Compared with the 3-

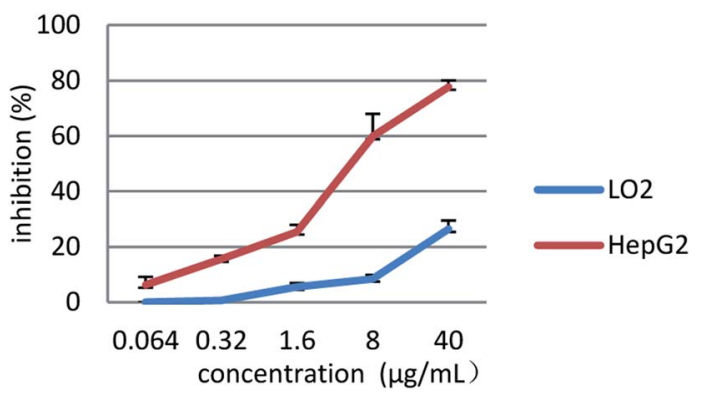

Fig. 6 Cytotoxicity of compound 4a toward HepG2 and LO2 cells. Data are means \pm SD of the inhibition (\%) from three independent experiments. 
Table 3 Kinase activities of compound $4 a$ and $4 d$

\begin{tabular}{lll}
\hline Entry & $\mathrm{ALK} / \mathrm{IC}_{50}(\mathrm{nM})$ & $\mathrm{c}-\mathrm{met} / \mathrm{IC}_{50}(\mathrm{nM})$ \\
\hline 4a & 203.56 & $>1000$ \\
4d & 686.19 & $>1000$ \\
Crizotinib & 11.21 & 7.68
\end{tabular}

methylphenyl substituted analog analogs 4d, the 3,4-methoxyphenyl substituted analog $\mathbf{4 a}$ were slightly more potent against ALK. In contrast, the two compounds exerted relatively weak inhibitory effect on c-MET/HGFR. As revealed from the results, the inhibition of ALK could be a mechanism for the antitumor effect of these novel carbamate derivatives.

\subsection{Molecular docking study}

As in a previously published co-crystal structure of Crizotinib with anaplastic lymphoma kinase (ALK), ${ }^{22}$ the 2-aminopyrimidine ring of Crizotinib formed two hydrogen bonds to the GLU1197 and MET1199 of the kinase hinge region. The lengths of the two hydrogen bonds were 1.87228 and $3.02638 \AA$, respectively. According to this model, the benzene ring of Crizotinib formed a $\mathrm{CH}-\pi$ interaction with the LEU1256, as well as a cation- $\pi$ bond with the Lys1150.

Like Crizotinib (Fig. 7), the compound 4a possessed a piperazine amide tail fragment can be easily docked into the ATP site of the DFG-out ALK co-crystal structure (PDB code: 2XP2). The docking conformation revealed that the 23,24dimethylcyclohexan-3-ol fragment of compound 4a was fully buried into the ATP binding site via hydrophobic interactions, compared with the pyridin-2-ylamine fragment of Crizotinib. Nevertheless, for its large size of the linker and the tail fragment, the compound $\mathbf{4 a}$ failed to form the expected hydrogen bonding interactions with the kinase hinge region. Moreover, the 3,4-dimethoxybenzoyl fragment formed a $\mathrm{CH}-\pi$ interaction with the ARG1120. The C3-OH of compound 4a pointed to the activation loop (DFG-out conformation). The compound 4 a with moderate enzymatic activity was relatively weakly bound to the

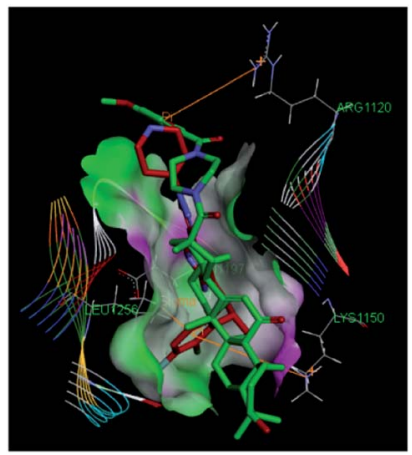

Fig. 7 Representative compound 4 a (green) and Crizotinib (red) in the active site of wild-type ALK.

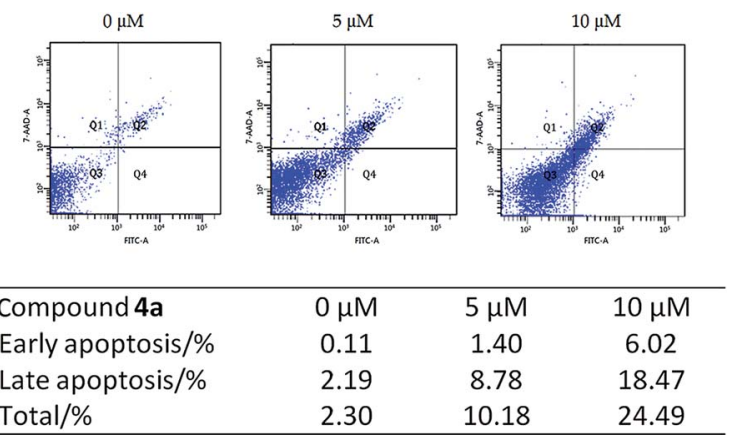

Fig. 8 Compound $4 \mathrm{a}$ induced apoptosis of HepG2 cells in a concentration-dependent manner.

potential binding sites, probably due to the large steric hindrance tail groups.

\subsection{Apoptosis detection by flow cytometry}

The effect of compound $\mathbf{4 a}$ on the apoptosis was investigated by means of a the 7-amino-actinomycin D (7-AAD) and annexin VFITC biparametric cytofluorimetric analysis. After treatment with different concentrations of compound $4 \mathbf{a}(0 \mu \mathrm{M}, 5 \mu \mathrm{M}, 10$ $\mu \mathrm{M})$ in serum-free medium for $48 \mathrm{~h}$, HepG2 cells were stained with 7-AAD and FITC, and then analyzed by the flow cytometry. As illustrated in Fig. 8, the percentage of total apoptotic cells from 10.18 to $24.49 \%$ was markedly elevated in a concentrationdependent manner, as compared to $2.30 \%$ for the control group.

\section{Conclusions}

In brief, a series of novel amide-linked 18 $\beta$-GA derivatives was synthesized and evaluated for their anticancer activity against Karpas299, A549, HepG2, MCF-7 and PC-3 cells. Of the compounds screened, some compounds with electron-donating groups on phenyl moiety exhibited evident antiproliferative activity. The most active compound 4a exhibited promising cytotoxicity against Karpas299 ( $\mathrm{IC}_{50}$ of $6.51 \mu \mathrm{M}$ ) and HepG2 $\left(\mathrm{IC}_{50}\right.$ of $\left.6.93 \mu \mathrm{M}\right)$ cells. Moreover, the compound $4 \mathrm{a}$ exhibited moderate inhibitory activity against wild-type ALK with $\mathrm{IC}_{50}$ value of $203.56 \mathrm{nM}$ and relatively weak potent to c-Met $\left(\mathrm{IC}_{50}>\right.$ $1000 \mathrm{nM}$ ). Furthermore, compound 4a induced apoptosis of HepG2 cells in a concentration-dependent manner, in particular at the late stage of the apoptotic process. Molecular docking was performed to delve into the differences in bonding modes between the compound $\mathbf{4 a}$ and Crizotinib. Lastly, the compound 4a would inspire further derivatization and optimization of such scaffold to explore more potent ALK inhibitors.

\section{Materials and general methods}

\subsection{Chemistry}

Unless otherwise required, all reagents used in the experiment were purchased as commercial analytical grade and used without further purification. Melting points were obtained in open capillary tubes with a WRS-1B melting point apparatus 
(Shanghai Shenguang Instrument Co., Ltd., Shanghai, CHN) and were uncorrected. The structure of the synthetic compound was confirmed by ${ }^{1} \mathrm{H}-\mathrm{NMR}$ and ${ }^{13} \mathrm{C}-\mathrm{NMR}$ spectra on $400 / 54$ Premium Shielded NMR Magnet System (Agilent, Santa Clara, USA) with tetramethylsilane (TMS) as an internal standard. HRMS spectra data were collected from an Agilent 6200 Series TOF and 6500 Series Q-TOF LC/MS System B.05.01. (B5125) in positive ion modes (Agilent, Santa Clara, USA).

tert-Butyl

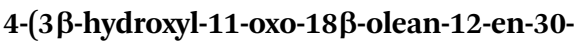
carbonyl)piperazine-1-carboxylate (2). 18ß-GA (1) (0.47 g, 1.0 $\mathrm{mmol})$ was dissolved in acetonitrile $(20 \mathrm{~mL})$, then $\mathrm{EDCl}(0.23 \mathrm{~g}$, $1.2 \mathrm{mmol})$, triethylamine $(0.13 \mathrm{~g}, 1.2 \mathrm{mmol})$ and HOBt $(0.16 \mathrm{~g}$, $1.2 \mathrm{mmol}$ ) were added. The mixture was stirred at room temperature for $20 \mathrm{~min}$. The 1-Boc-piperazine $(0.22 \mathrm{~g}, 1.2 \mathrm{mmol})$ was added, and the mixture was stirred under reflux for $24 \mathrm{~h}$. The solvent was removed under vacuum to give a residue which was treated with a mixture of ethanol and water. The solution was stirred at room temperature for $30 \mathrm{~min}$, and a solid was obtained by filtration while washing with $\mathrm{H}_{2} \mathrm{O}$.

A white solid; yield, 94.3\%; mp 224.3-225.7 ${ }^{\circ} \mathrm{C} ;{ }^{1} \mathrm{H}$ NMR $(400$ $\mathrm{MHz}$, chloroform- $d$ ) $\delta 5.66(\mathrm{~s}, 1 \mathrm{H}, \mathrm{CH}-12), 3.63-3.52(\mathrm{~m}, 4 \mathrm{H}$, piperazinyl $\left.\mathrm{CH}_{2} \times 2\right), 3.39\left(\mathrm{t}, J=5.2 \mathrm{~Hz}, 4 \mathrm{H}\right.$, piperazinyl $\mathrm{CH}_{2} \times$ 2), 3.22-3.18 (m, 1H, OH-3), 2.79-2.74 (m, 1H, CH-1), 2.31 (s, 1H, CH-9), 2.30-2.23 (m, 1H, CH-16), 1.45 (s, 9H, tert-butyl $\mathrm{CH}_{3}$ $\times 3), 1.34\left(\mathrm{~s}, 3 \mathrm{H}, \mathrm{CH}_{3}-27\right), 1.20\left(\mathrm{~s}, 3 \mathrm{H}, \mathrm{CH}_{3}-25\right), 1.11\left(\mathrm{~s}, 3 \mathrm{H}, \mathrm{CH}_{3}-\right.$ 26), 1.10 (s, 3H, $\left.\mathrm{CH}_{3}-29\right), 0.98$ (s, 3H, $\left.\mathrm{CH}_{3}-23\right), 0.79$ (s, 3H, $\mathrm{CH}_{3^{-}}$ 24), 0.78 (s, 3H, $\left.\mathrm{CH}_{3}-28\right), 0.68$ (d, $\left.J=11.6 \mathrm{~Hz}, 1 \mathrm{H}, \mathrm{CH}-5\right) ;{ }^{13} \mathrm{C}$ NMR (101 MHz, chloroform- $d$ ) $\delta 200.10$ (C11), 174.13 (C30), 169.40 (C13), 154.53 (Boc $\mathrm{C}=\mathrm{O}$ ), 128.56 (C12), 80.25 (C3), 78.75 (tert-butyl C), 61.77 (C9), 54.92 (C5), 48.08 (C18), 45.26 (C14), 43.88 (C20), 43.82 (piperazinyl C $\times 2$ ), 43.26 (C8/C19), 39.12 (C1/ C4), 39.10 (piperazinyl $\mathrm{C} \times 2$ ), 37.70 (C22), 37.06 (C10), 33.16 (C7), 32.79 (C17), 31.75 (C21), 28.40 (C29), 28.36 (tert-butyl $\mathrm{CH}_{3}$ $\times$ 3), 28.07 (C28), 27.28 (C23), 27.05 (C2), 26.69 (C15), 26.39 (C16), 23.14 (C27), 18.66 (C26), 17.46 (C6), 16.36 (C25), 15.56 (C24); HRMS (m/z): $[\mathrm{M}+\mathrm{H}]^{+}$calcd for $\mathrm{C}_{39} \mathrm{H}_{63} \mathrm{~N}_{2} \mathrm{O}_{5}$ : 639.47370, found: 639.47360 .

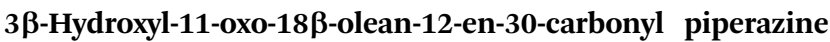
(3). Compound 2 (0.64 g, $1.0 \mathrm{mmol})$ was dissolved in $\mathrm{CH}_{2} \mathrm{Cl}_{2}(10$ $\mathrm{mL})$ at $0{ }^{\circ} \mathrm{C}$ under stirring. Trifluoroacetic acid $(5 \mathrm{~mL})$ was added, and the reaction was stirred at $0{ }^{\circ} \mathrm{C}$ for $3 \mathrm{~h}$. After reaction, the mixture was partitioned between $\mathrm{CH}_{2} \mathrm{Cl}_{2}$ and saturated aqueous $\mathrm{NaHCO}_{3}$. The organic phase was washed with water, dried over $\mathrm{Na}_{2} \mathrm{SO}_{4}$, and concentrated to give the desired product.

A white solid; yield, 97.0\%; mp 258.5-259.7 ${ }^{\circ} \mathrm{C}$ (literature (ref. 23): $160{ }^{\circ} \mathrm{C}$, decomp.); ${ }^{1} \mathrm{H}$ NMR (400 MHz, chloroform- $d$ ) $\delta 5.67(\mathrm{~s}, 1 \mathrm{H}, \mathrm{CH}-12), 3.63\left(\mathrm{q}, J=3.7 \mathrm{~Hz}, 4 \mathrm{H}\right.$, piperazinyl $\mathrm{CH}_{2} \times$ 2), $3.20(\mathrm{dd}, J=10.9,5.4 \mathrm{~Hz}, 1 \mathrm{H}, \mathrm{OH}-3), 2.88(\mathrm{t}, J=5.0 \mathrm{~Hz}, 4 \mathrm{H}$, piperazinyl $\left.\mathrm{CH}_{2} \times 2\right), 2.76(\mathrm{dt}, J=13.6,3.6 \mathrm{~Hz}, 1 \mathrm{H}, \mathrm{CH}-1), 2.31$ (s, 1H, CH-9), 2.26 (dd, $J=14.0,3.9 \mathrm{~Hz}, 1 \mathrm{H}, \mathrm{CH}-16), 1.36$ (s, 3H, $\left.\mathrm{CH}_{3}-27\right), 1.19$ (s, 3H, $\left.\mathrm{CH}_{3}-25\right), 1.11$ (s, 3H, $\left.\mathrm{CH}_{3}-26\right), 1.10$ (s, 3H, $\left.\mathrm{CH}_{3}-29\right), 0.98$ (s, 3H, $\left.\mathrm{CH}_{3}-23\right), 0.79$ (s, 3H, $\left.\mathrm{CH}_{3}-24\right), 0.78$ (s, 3H, $\left.\mathrm{CH}_{3}-28\right), 0.67$ (d, $\left.J=11.4 \mathrm{~Hz}, 1 \mathrm{H}\right) ;{ }^{13} \mathrm{C}$ NMR (101 MHz, chloroform- $d$ ) $\delta 200.19$ (C11), 173.89 (C30), 169.64 (C13), 128.51 (C12), 78.73 (C3), 61.77 (C9), 54.92 (C5), 48.16 (C18), 45.94 (piperazinyl $\mathrm{C} \times 2$ ), 45.26 (C14), 43.79 (C20), 43.27 (C8), 39.14 (piperazinyl C $\times 2$ ), 39.11 (C19), 37.72 (C1/4), 37.07 (C22), 33.28 (C10), 32.78 (C7), 31.76 (C17), 28.41 (C21), 28.07 (C29), 27.28 (C28), 27.00 (C23), 26.70 (C2), 26.42 (C15), 23.13 (C27), 18.66 (C26), 17.46 (C6), 16.37 (C25), 15.57 (C24); HRMS (m/z): [M + H $]^{+}$calcd for $\mathrm{C}_{34} \mathrm{H}_{55} \mathrm{~N}_{2} \mathrm{O}_{3}$ : 539.42127, found: 539.42120.

General procedure for preparation of carbamate derivatives (4a-4y). Compound $3(0.54 \mathrm{~g}, 1.0 \mathrm{mmol})$ and triethylamine $(0.13 \mathrm{~g}, 1.2 \mathrm{mmol})$ were dissolved in $\mathrm{CH}_{2} \mathrm{Cl}_{2}(20 \mathrm{~mL})$ at $0{ }^{\circ} \mathrm{C}$ under stirring. Substituted acyl chloride $(1.0 \mathrm{mmol})$ was added, and the reaction was stirred at room temperature. After reaction, the mixture was washed with saturated aqueous $\mathrm{NaHCO}_{3}$ and water. The organic layer was dried over anhydrous $\mathrm{Na}_{2} \mathrm{SO}_{4}$, filtered, and concentrated under reduced pressure. The crude product was then chromatographed on silica $\left(20: 1 \mathrm{CH}_{2} \mathrm{Cl}_{2}-\right.$ methanol).

3 $\beta$-Hydroxy-30-(4-(3,4-dimethoxybenzoyl)-1-piperazinyl)-olean12-ene-11,30-dione (4a). A white solid; yield, 94.2\%; mp 221.9$222.8{ }^{\circ} \mathrm{C}$; ${ }^{1} \mathrm{H}$ NMR (400 MHz, chloroform- $\left.d\right) \delta 7.01(\mathrm{~s}, 2 \mathrm{H}$, phenyl-H), 6.88 (s, 1H, phenyl-H), 5.68 (s, 1H, CH-12), 3.92 (s, $\left.6 \mathrm{H},-\mathrm{OCH}_{3}\right), 3.66(\mathrm{~s}, 8 \mathrm{H}$, piperazinyl-H), 3.23 (dd, $J=10.8$, $5.2 \mathrm{~Hz}, 1 \mathrm{H}, 3-\mathrm{OH}), 2.78$ (d, $J=13.2 \mathrm{~Hz}, 1 \mathrm{H}, \mathrm{CH}-1), 2.34$ (s, 1H, $\mathrm{CH}-9$ ), 2.27 (d, $J=12.8 \mathrm{~Hz}, 1 \mathrm{H}, \mathrm{CH}-16$ ), 1.36 (s, 3H, $\left.\mathrm{CH}_{3}-27\right), 1.25$ (s, 3H, $\left.\mathrm{CH}_{3}-25\right), 1.14$ (s, 3H, $\left.\mathrm{CH}_{3}-26\right), 1.12$ (s, 3H, $\left.\mathrm{CH}_{3}-29\right), 1.01$ (s, 3H, $\left.\mathrm{CH}_{3}-23\right), 0.82$ (s, 3H, $\left.\mathrm{CH}_{3}-24\right), 0.81$ (s, 3H, $\left.\mathrm{CH}_{3}-28\right), 0.70$ $(\mathrm{d}, J=11.4 \mathrm{~Hz}, 1 \mathrm{H}, \mathrm{CH}-5) ;{ }^{13} \mathrm{C}$ NMR $(101 \mathrm{MHz}$, chloroform- $d$ ) $\delta 200.07$ (C11), 174.24 (C30), 170.50 (C13), 169.32 (benzoyl, $\mathrm{C}=\mathrm{O}$ ), 150.52 (phenyl), 149.04 (phenyl), 128.55 (C12), 127.28 (phenyl), 120.19 (phenyl) 110.85 (phenyl), 110.46 (phenyl), 78.72 (C3), 61.78 (C9), $56.01\left(-\mathrm{OCH}_{3}\right), 55.99\left(-\mathrm{OCH}_{3}\right), 54.91$ (C5), 48.19 (C18), 45.27 (C14), 43.90 (C20), 43.72 (C8), 43.27 (piperazinyl C), 39.10 (piperazinyl C), 37.68 (C4), 37.05 (C22), 33.26 (C10), 32.78 (C7), 31.79 (C17), 29.69 (C21), 28.38 (C29), 28.07 (C28), 27.27 (C23), 27.06 (C2), 26.68 (C15), 26.40 (C16), 23.15 (C27), 18.66 (C26), 17.46 (C6), 16.37 (C25), 15.57 (C24); HRMS (m/z): [M + $\mathrm{Na}]^{+}$calcd for $\mathrm{C}_{43} \mathrm{H}_{62} \mathrm{~N}_{2} \mathrm{NaO}_{6}$ : 725.45056, found: 725.45778 .

3 $\beta$-Hydroxy-30-(4-(3,5-dimethoxybenzoyl)-1-piperazinyl)-olean12-ene-11,30-dione (4b). A white solid; yield, 92.5\%; mp 224.1$226.0{ }^{\circ} \mathrm{C} ;{ }^{1} \mathrm{H}$ NMR (400 MHz, chloroform- $d$ ) $\delta$ 6.55-6.47 (m, 3H, phenyl-H), 5.68 (s, 1H, CH-12), 3.81 (s, 6H, phenyl- $\mathrm{CH}_{3}$ ), 3.743.39 (m, 8H, piperazinyl-H), 3.22 (dd, $J=10.8,5.4 \mathrm{~Hz}, 1 \mathrm{H}, 3-$ $\mathrm{OH}), 2.78$ (dt, $J=13.4,3.5 \mathrm{~Hz}, 1 \mathrm{H}, \mathrm{CH}-1), 2.33$ (s, 1H, CH-9), 2.28 (d, $J=12.6 \mathrm{~Hz}, 1 \mathrm{H}, \mathrm{CH}-16), 1.36$ (s, 3H, $\left.\mathrm{CH}_{3}-27\right), 1.23$ (s, 3H, $\left.\mathrm{CH}_{3}-25\right), 1.14$ (s, 3H, $\left.\mathrm{CH}_{3}-26\right), 1.12$ (s, 3H, $\left.\mathrm{CH}_{3}-29\right), 1.00$ (s, 3H, $\left.\mathrm{CH}_{3}-23\right), 0.82$ (s, 3H, $\left.\mathrm{CH}_{3}-24\right), 0.81$ (s, 3H, $\left.\mathrm{CH}_{3}-28\right), 0.70$ (d, $J=$ $11.8 \mathrm{~Hz}, 1 \mathrm{H}, \mathrm{CH}-5) ;{ }^{13} \mathrm{C}$ NMR (101 MHz, chloroform-d) $\delta 200.05$ (C11), 174.22 (C30), 170.16 (C13), 169.27 (benzoyl, C=O), 160.91 (phenyl), 137.02 (phenyl), 128.56 (C12), 104.77 (phenyl), 101.90 (phenyl), 78.73 (C3), 61.77 (C9), $55.51\left(-\mathrm{OCH}_{3}\right), 54.91$ (C5), 48.13 (C18), 45.26 (C14), 43.89 (C20), 43.75 (C8), 43.26 (piperazinyl C), 39.10 (piperazinyl C), 37.67 (C22), 37.05 (C10), 33.18 (C7), 32.78 (C17), 31.78 (C21), 28.38 (C29), 28.07 (C28), 27.28 (C23), 27.04 (C2), 26.67 (C15), 26.39 (C16), 23.14 (C27), 18.65 (C26), 17.46 (C6), 16.37 (C25), 15.56 (C24); HRMS (m/z): [M + H $]^{+}$calcd for $\mathrm{C}_{43} \mathrm{H}_{63} \mathrm{~N}_{2} \mathrm{O}_{6}$ : 703.46861, found: 703.47552.

3 $\beta$-Hydroxy-30-(4-(3,5-dimethylbenzoyl)-1-piperazinyl)-olean12-ene-11,30-dione (4c). A white solid; yield, 92.7\%; mp 232.4- 
232.7 ${ }^{\circ} \mathrm{C} ;{ }^{1} \mathrm{H}$ NMR (400 MHz, chloroform- $d$ ) $\delta 7.06(\mathrm{~s}, 1 \mathrm{H}$, phenyl-H), 7.00 (s, 2H, phenyl-H), 5.68 (s, 1H, CH-12), 3.57 (m, $8 \mathrm{H}$, piperazinyl-H), 3.22 (dd, $J=10.8,5.4 \mathrm{~Hz}, 1 \mathrm{H}, 3-\mathrm{OH}), 2.78$ (dt, $J=13.5,3.5 \mathrm{~Hz}, 1 \mathrm{H}, \mathrm{CH}-1), 2.34$ (s, 6H, phenyl- $\left.\mathrm{CH}_{3}\right), 2.29$ (d, $J=13.1 \mathrm{~Hz}, 1 \mathrm{H}, \mathrm{CH}-16), 1.36$ (s, 3H, $\left.\mathrm{CH}_{3}-27\right), 1.23$ (s, 3H, $\mathrm{CH}_{3}-$ 25), 1.14 (s, 3H, $\left.\mathrm{CH}_{3}-26\right), 1.12$ (s, 3H, $\left.\mathrm{CH}_{3}-29\right), 1.00$ (s, 3H, $\mathrm{CH}_{3}-$ 23), 0.82 (s, 3H, $\left.\mathrm{CH}_{3}-24\right), 0.81$ (s, 3H, $\left.\mathrm{CH}_{3}-28\right), 0.70$ (d, $J=$ 11.5 Hz, 1H, CH-5); ${ }^{13} \mathrm{C}$ NMR (101 MHz, chloroform- $d$ ) $\delta 200.06$ (C11), 174.22 (C30), 170.93 (C13), 169.27 (benzoyl, C=O), 138.34 (phenyl), 135.13 (phenyl), 131.52 (phenyl), 128.59 (C12), 124.59 (phenyl), 78.75 (C3), 61.78 (C9), 54.92 (C5), 48.10 (C18), 45.27 (C14), 43.90 (C20), 43.78 (C8), 43.26 (piperazinyl C), 39.11 (piperazinyl C), 37.69 (C22), 37.06 (C10), 33.15 (C7), 32.79 (C17), 31.77 (C21), 28.39 (C29), 28.07 (C28), 27.29 (C23), 27.06 (C2), 26.69 (C15), 26.39 (C16), 23.14 (C27), 21.26 (phenyl- $\mathrm{CH}_{3}$ ), 18.66 (C26), 17.47 (C6), 16.37 (C25), 15.56 (C24); HRMS (m/z): [M + $\mathrm{Na}]^{+}$calcd for $\mathrm{C}_{43} \mathrm{H}_{63} \mathrm{~N}_{2} \mathrm{O}_{4}: 671.47878$, found: 671.48498 .

3ß-Hydroxy-30-(4-(3-methylbenzoyl)-1-piperazinyl)-olean-12ene-11,30-dione (4d). A white solid; yield, 91.5\%; $\mathrm{mp} 263.4-$ $265.8{ }^{\circ} \mathrm{C} ;{ }^{1} \mathrm{H}$ NMR $(400 \mathrm{MHz}$, chloroform- $d$ ) $\delta 7.35-7.14(\mathrm{~m}, 4 \mathrm{H}$, phenyl-H), 5.68 (s, 1H, CH-12), 3.88-3.35 (m, 8H, piperazinyl- $\mathrm{H}$ ), $3.22(\mathrm{dd}, J=10.8,5.4 \mathrm{~Hz}, 1 \mathrm{H}, 3-\mathrm{OH}), 2.78(\mathrm{dt}, J=13.5,3.5 \mathrm{~Hz}$, $1 \mathrm{H}, \mathrm{CH}-1), 2.38$ (s, 3H, phenyl- $\left.\mathrm{CH}_{3}\right), 2.33$ (s, 1H, CH-9), 2.29 (d, $=10.7 \mathrm{~Hz}, 1 \mathrm{H}, \mathrm{CH}-16), 1.36\left(\mathrm{~s}, 3 \mathrm{H}, \mathrm{CH}_{3}-27\right), 1.23\left(\mathrm{~s}, 3 \mathrm{H}, \mathrm{CH}_{3}-25\right)$, 1.14 (s, 3H, $\left.\mathrm{CH}_{3}-26\right), 1.12$ (s, 3H, $\left.\mathrm{CH}_{3}-29\right), 1.00$ (s, 3H, $\left.\mathrm{CH}_{3}-23\right)$, 0.82 (s, $\left.3 \mathrm{H}, \mathrm{CH}_{3}-24\right), 0.81$ (s, 3H, $\left.\mathrm{CH}_{3}-28\right), 0.70$ (d, $J=11.6 \mathrm{~Hz}$, $1 \mathrm{H}, \mathrm{CH}-5) ;{ }^{13} \mathrm{C}$ NMR (101 MHz, chloroform- $d$ ) $\delta 200.05$ (C11), 174.22 (C30), 170.76 (C13), 169.26 (benzoyl, C=O), 138.59 (phenyl), 135.11 (phenyl), 130.72 (phenyl), 128.59 (C12), 128.41 (phenyl), 127.70 (phenyl), 123.95 (phenyl), 78.75 (C3), 61.78 (C9), 54.92 (C5), 48.10 (C18), 45.26 (C14), 43.91 (C20), 43.78 (C8), 43.26 (piperazinyl C), 39.11 (piperazinyl C), 37.69 (C1/4), 37.06 (C22), 33.15 (C10), 32.79 (C7), 31.77 (C17), 29.69 (C21), 28.39 (C29), 28.07 (C28), 27.29 (C23), 27.05 (C2), 26.69 (C15), 26.39 (C16), 23.14 (C27), 21.37 (phenyl $\mathrm{CH}_{3}$ ), 18.66 (C26), 17.47 (C6), 16.37 (C25), 15.56 (C24); HRMS $(\mathrm{m} / \mathrm{z}):[\mathrm{M}+\mathrm{H}]^{+}$calcd for $\mathrm{C}_{42} \mathrm{H}_{61} \mathrm{~N}_{2} \mathrm{O}_{4}$ : 657.46313, found: 657.46856 .

3 $\beta$-Hydroxy-30-(4-(3,4-dichlorobenzoyl)-1-piperazinyl)-olean-12ene-11,30-dione (4e). A white solid; yield, 93.4\%; mp 237.9$239.6{ }^{\circ} \mathrm{C} ;{ }^{1} \mathrm{H}$ NMR $(400 \mathrm{MHz}$, chloroform- $d$ ) $\delta 7.52(\mathrm{~d}, J=8.5 \mathrm{~Hz}$, 2H, phenyl-H), 7.29-7.24 (m, 2H, phenyl-H), 5.67 (s, 1H, CH-12), 3.68-3.47 (m, 8H, piperazinyl-H), $3.22(\mathrm{dd}, J=11.0,5.2 \mathrm{~Hz}, 1 \mathrm{H}$, 3-OH), 2.78 (d, $J=13.2 \mathrm{~Hz}, 1 \mathrm{H}, \mathrm{CH}-1$ ), 2.33 (s, 1H, CH-9), 2.27 (d, $J=13.0 \mathrm{~Hz}, 1 \mathrm{H}, \mathrm{CH}-16), 1.36\left(\mathrm{~s}, 3 \mathrm{H}, \mathrm{CH}_{3}-27\right), 1.24$ (s, 3H, $\mathrm{CH}_{3}-$ 25), 1.14 (s, 3H, $\left.\mathrm{CH}_{3}-26\right), 1.12$ (s, 3H, $\left.\mathrm{CH}_{3}-29\right), 1.00$ (s, 3H, $\mathrm{CH}_{3}-$ 23), 0.82 (s, 3H, $\left.\mathrm{CH}_{3}-24\right), 0.81$ (s, 3H, $\left.\mathrm{CH}_{3}-28\right), 0.70$ (d, $J=$ $11.5 \mathrm{~Hz}, 1 \mathrm{H}, \mathrm{CH}-5) ;{ }^{13} \mathrm{C}$ NMR (101 MHz, chloroform- $d$ ) $\delta 200.09$ (C11), 174.28 (C30), 169.24 (C13), 168.09 (benzoyl, C=O), 134.82 (phenyl), 134.52 (phenyl), 133.19 (phenyl), 130.75 (phenyl), 129.36 (phenyl), 128.58 (C12), 126.42 (phenyl), 78.74 (C3), 61.79 (C9), 54.92 (C5), 48.14 (C18), 45.27 (C14), 43.92 (C20), 43.74 (C8), 43.27 (piperazinyl C), 39.11 (piperazinyl C), 37.67 (C4), 37.06 (C22), 33.19 (C10), 32.79 (C7), 31.79 (C17), 29.69 (C21), 28.39 (C29), 28.07 (C28), 27.39 (C23), 27.05 (C2), 26.67 (C15), 26.39 (C16), 23.15 (C27), 18.66 (C26), 17.45 (C6), 16.37 (C25), 15.56 (C24); HRMS $(\mathrm{m} / \mathrm{z}):[\mathrm{M}+\mathrm{Na}]^{+}$calcd for $\mathrm{C}_{41} \mathrm{H}_{56} \mathrm{Cl}_{2} \mathrm{~N}_{2} \mathrm{NaO}_{4}$ : 733.35148, found: 733.35752 .
3 $\beta$-Hydroxy-30-(4-(2,4-dichlorobenzoyl)-1-piperazinyl)-olean-12ene-11,30-dione (4f). A white solid; yield, 93.4\%; mp 231.9$233.3{ }^{\circ} \mathrm{C} ;{ }^{1} \mathrm{H}$ NMR (400 MHz, chloroform- $d$ ) $\delta 7.45(\mathrm{~s}, 1 \mathrm{H}$, phenyl-H), 7.34 (d, $J=8.3 \mathrm{~Hz}, 1 \mathrm{H}$, phenyl-H), $7.24(\mathrm{~s}, 1 \mathrm{H}$, phenyl-H), 5.67 (d, $J=5.3 \mathrm{~Hz}, 1 \mathrm{H}, \mathrm{CH}-12), 3.99-3.54(\mathrm{~m}, 6 \mathrm{H}$, piperazinyl-H), 3.34-3.23 (m, 2H, piperazinyl- $\mathrm{H}), 3.21$ (d, $J=$ $5.3 \mathrm{~Hz}, 1 \mathrm{H}, 3-\mathrm{OH}), 2.82-2.74$ (m, 1H, CH-1), 2.34 (s, 1H, CH-9), 2.27 (d, $J=13.4 \mathrm{~Hz}, 1 \mathrm{H}, \mathrm{CH}-16), 1.36$ (s, 3H, $\left.\mathrm{CH}_{3}-27\right), 1.23$ (s, 3H, $\left.\mathrm{CH}_{3}-25\right), 1.14$ (s, 3H, $\left.\mathrm{CH}_{3}-26\right), 1.12$ (s, 3H, $\left.\mathrm{CH}_{3}-29\right), 1.00(\mathrm{~s}, 3 \mathrm{H}$, $\left.\mathrm{CH}_{3}-23\right), 0.82$ (s, 3H, $\left.\mathrm{CH}_{3}-24\right), 0.81$ (s, 3H, $\left.\mathrm{CH}_{3}-28\right), 0.70$ (d, $J=$ $11.5 \mathrm{~Hz}, 1 \mathrm{H}, \mathrm{CH}-5) ;{ }^{13} \mathrm{C}$ NMR (101 MHz, chloroform-d) $\delta 200.09$ (C11), 174.23 (C30), 169.29 (C13), 166.11 (benzoyl, C=O), 135.90 (phenyl), 133.64 (phenyl), 131.20 (phenyl), 129.68 (phenyl), 128.87 (phenyl), 128.80 (phenyl), 128.59 (C12), 127.85 (phenyl), 78.75 (C3), 61.78 (C9), 54.92 (C5), 48.10 (C18), 46.76 (C14), 45.27 (C20), 43.91 (C8), 43.26 (piperazinyl C), 41.88 (C19), 39.10 (piperazinyl C), 37.68 (C4), 37.05 (C22), 33.26 (C10), 32.78 (C7), 31.79 (C17), 29.69 (C21), 28.38 (C29), 28.07 (C28), 27.27 (C23), 27.06 (C2), 26.68 (C15), 26.40, 41.88 (piperazinyl C), 39.11 (C4), 37.67 (C22), 37.06 (C10), 32.79 (C7), 31.77 (C17), 29.69 (C21), 28.38 (C29), 28.07 (C28), 27.28 (C23), 27.06 (C2), 26.67 (C15), 26.38 (C16), 23.15 (C27), 18.65 (C26), 17.46 (C6), 16.37 (C25), 15.56 (C24); HRMS (m/z): $[\mathrm{M}+\mathrm{H}]^{+}$calcd for $\mathrm{C}_{41} \mathrm{H}_{57} \mathrm{Cl}_{2} \mathrm{~N}_{2} \mathrm{O}_{4}$ : 711.36954, found: 711.37585 .

3 $\beta$-Hydroxy-30-(4-(3,5-dichlorobenzoyl)-1-piperazinyl)-olean-12ene-11,30-dione (4g). A white solid; yield, 92.9\%; mp 235.5$236.9{ }^{\circ} \mathrm{C} ;{ }^{1} \mathrm{H}$ NMR $(400 \mathrm{MHz}$, chloroform- $d$ ) $\delta 7.44(\mathrm{t}, J=1.9 \mathrm{~Hz}$, $1 \mathrm{H}$, phenyl-H), 7.29 (d, $J=1.9 \mathrm{~Hz}, 2 \mathrm{H}$, phenyl-H), $5.68(\mathrm{~s}, 1 \mathrm{H}$, $\mathrm{CH}-12$ ), 3.74-3.41 (m, 8H, piperazinyl-H), 3.22 (dd, $J=10.7$, $5.5 \mathrm{~Hz}, 1 \mathrm{H}, 3-\mathrm{OH}), 2.83-2.74$ (m, 1H, CH-1), 2.33 (s, 1H, CH-9), $2.27(\mathrm{~d}, J=12.7 \mathrm{~Hz}, 1 \mathrm{H}, \mathrm{CH}-16), 1.36$ (s, 3H, $\left.\mathrm{CH}_{3}-27\right), 1.24(\mathrm{~s}, 3 \mathrm{H}$, $\left.\mathrm{CH}_{3}-25\right), 1.14$ (s, 3H, $\left.\mathrm{CH}_{3}-26\right), 1.12$ (s, 3H, $\left.\mathrm{CH}_{3}-29\right), 1.00$ (s, 3H, $\left.\mathrm{CH}_{3}-23\right), 0.82$ (s, 3H, $\left.\mathrm{CH}_{3}-24\right), 0.81$ (s, 3H, $\left.\mathrm{CH}_{3}-28\right), 0.70$ (d, $J=$ $11.5 \mathrm{~Hz}, 1 \mathrm{H}, \mathrm{CH}-5) ;{ }^{13} \mathrm{C}$ NMR (101 MHz, chloroform-d) $\delta 199.91$ (C11), 174.27 (C30), 171.02 (C13), 169.28 (benzoyl, C=O), 167.78 (C-Cl), 164.23 (C-Cl), 164.11 (C-Cl), 161.73 (C-Cl), 161.61 (C$\mathrm{Cl}$ ), 138.13 (phenyl), 128.53 (C12), 110.54 (phenyl), 110.46 (phenyl), 110.35 (phenyl), 110.27 (phenyl), 105.83 (phenyl), 105.58 (phenyl), 105.33 (phenyl), 80.55 (C3), 61.69 (C9), 54.99 (C5), 48.15 (C18), 45.28 (C14), 43.92 (C20), 43.70 (C8), 43.26 (piperazinyl C), 38.77 (piperazinyl C), 38.01 (C4), 37.66 (C22), 36.90 (10), 33.19 (C7), 32.72 (C17), 31.78 (C21), 29.69 (C29), 28.39 (C28), 28.02 (C23), 27.04 (C2), 26.66 (C15), 26.36 (C16), 21.32 (C27), 18.65 (C26), 17.34 (C6), 16.66 (C25), 16.41 (C24); HRMS $(\mathrm{m} / \mathrm{z}):[\mathrm{M}+\mathrm{Na}]^{+}$calcd for $\mathrm{C}_{41} \mathrm{H}_{56} \mathrm{Cl}_{2} \mathrm{~N}_{2} \mathrm{NaO}_{4}: 733.35148$, found: 733.35754 .

3 $\beta$-Hydroxy-30-(4-(3-chlorobenzoyl)-1-piperazinyl)-olean-12-

ene-11,30-dione (4h). A white solid; yield, 92.1\%; $\mathrm{mp} 222.9-$ $224.0{ }^{\circ} \mathrm{C} ;{ }^{1} \mathrm{H}$ NMR (400 MHz, chloroform- $d$ ) $\delta 7.46-7.33(\mathrm{~m}, 3 \mathrm{H}$, phenyl-H), 7.29 (dd, $J=7.3,1.7 \mathrm{~Hz}, 1 \mathrm{H}$, phenyl-H), $5.68(\mathrm{~s}, 1 \mathrm{H}$, CH-12), 3.74-3.44 (m, 8H, piperazinyl-H), 3.23 (dd, $J=10.8$, $5.5 \mathrm{~Hz}, 1 \mathrm{H}, 3-\mathrm{OH}), 2.81-2.76$ (m, 1H, CH-1), 2.34 (s, 1H, CH-9), $2.28(\mathrm{~d}, J=11.5 \mathrm{~Hz}, 1 \mathrm{H}, \mathrm{CH}-16), 1.36$ (s, 3H, $\left.\mathrm{CH}_{3}-27\right), 1.24(\mathrm{~s}, 3 \mathrm{H}$, $\left.\mathrm{CH}_{3}-25\right), 1.14$ (s, 3H, $\left.\mathrm{CH}_{3}-26\right), 1.12\left(\mathrm{~s}, 3 \mathrm{H}, \mathrm{CH}_{3}-29\right), 1.01(\mathrm{~s}, 3 \mathrm{H}$, $\left.\mathrm{CH}_{3}-23\right), 0.82$ (s, 3H, $\left.\mathrm{CH}_{3}-24\right), 0.81$ (s, 3H, $\left.\mathrm{CH}_{3}-28\right), 0.70$ (d, $J=$ $11.6 \mathrm{~Hz}, 1 \mathrm{H}, \mathrm{CH}-5) ;{ }^{13} \mathrm{C}$ NMR (101 MHz, chloroform- $d$ ) $\delta 200.07$ 
(C11), 174.27 (C30), 169.26 (C13), 168.97 (benzoyl, C=O), 136.81 (phenyl), 134.76 (phenyl), 130.19 (phenyl), 130.02 (phenyl), 128.59 (C12), 127.31 (phenyl), 125.15 (phenyl), 78.75 (C3), 61.78 (C9), 54.92 (C5), 48.11 (C18), 45.27 (C14), 43.92 (C20), 43.77 (C8), 43.26 (piperazinyl C), 39.12 (piperazinyl C), 39.10 (C1), 37.68 (C4), 37.06 (C22), 33.15 (C10), 32.79 (C7), 31.78 (C17), 29.69 (C21), 28.39 (C29), 28.07 (C28), 27.28 (C23), 27.04 (C2), 26.68 (C15), 26.38 (C16), 23.14 (C27), 18.66 (C26), 17.46 (C6), 16.37 (C25), 15.56 (C24); HRMS (m/z): $[\mathbf{M}+\mathrm{H}]^{+}$calcd for $\mathrm{C}_{41} \mathrm{H}_{58} \mathrm{ClN}_{2} \mathrm{O}_{4}$ : 677.40851, found: 677.41461 .

3ß-Hydroxy-30-(4-(4-chlorobenzoyl)-1-piperazinyl)-olean-12ene-11,30-dione (4i). A white solid; yield, 94.0\%; $\mathrm{mp} 233.9-$ $234.6{ }^{\circ} \mathrm{C} ;{ }^{1} \mathrm{H}$ NMR $(400 \mathrm{MHz}$, chloroform- $d$ ) $\delta 7.39(\mathrm{~d}, J=$ 16.0 Hz, 3H, phenyl-H), 7.29 (d, $J=7.4 \mathrm{~Hz}, 1 \mathrm{H}$, phenyl-H), 5.68 (s, 1H, CH-12), 3.69 (s, 6H, piperazinyl-H), $3.45(\mathrm{~s}, 2 \mathrm{H}$, piperazinyl-H), 3.22 (dd, $J=10.8,5.3 \mathrm{~Hz}, 1 \mathrm{H}, 3-\mathrm{OH}), 2.78(\mathrm{dt}, J=$ 13.6, 3.5 Hz, 1H, CH-1), 2.33 (s, 1H, CH-9), 2.32-2.25 (m, 1H, $\mathrm{CH}-16), 1.36$ (s, 3H, $\left.\mathrm{CH}_{3}-27\right), 1.23$ (s, 3H, $\left.\mathrm{CH}_{3}-25\right), 1.14$ (s, 3H, $\left.\mathrm{CH}_{3}-26\right), 1.12$ (s, 3H, $\left.\mathrm{CH}_{3}-29\right), 1.00$ (s, 3H, $\left.\mathrm{CH}_{3}-23\right), 0.82$ (s, 3H, $\left.\mathrm{CH}_{3}-24\right), 0.81$ (s, 3H, $\left.\mathrm{CH}_{3}-28\right), 0.70(\mathrm{~d}, J=11.5 \mathrm{~Hz}, 1 \mathrm{H}, \mathrm{CH}-5) ;{ }^{13} \mathrm{C}$ NMR (101 MHz, chloroform-d) $\delta 200.05$ (C11), 174.26 (C30), 169.24 (C13), 168.95 (benzoyl, $\mathrm{C}=\mathrm{O}$ ), 136.82 (phenyl), 134.75 (phenyl), 130.18 (phenyl), 130.02 (phenyl), 128.58 (C12), 127.30 (phenyl), 125.14 (phenyl), 78.72 (C3), 61.78 (C9), 54.91 (C5), 48.10 (C18), 45.26 (C14), 43.91 (C20), 43.77 (C8), 43.26 (piperazinyl C), 39.11 (piperazinyl C), 37.67 (C4), 37.06 (C22), 33.14 (C10), 32.78 (C7), 31.77 (C17), 29.69 (C21), 28.39 (C29), 28.07 (C28), 27.28 (C23), 27.04 (C2), 26.67 (C15), 26.38 (C16), 23.14 (C27), 18.66 (C26), 17.46 (C6), 16.36 (C25), 15.57 (C24); HRMS $(\mathrm{m} / \mathrm{z}):[\mathrm{M}+\mathrm{H}]^{+}$calcd $\mathrm{C}_{41} \mathrm{H}_{58} \mathrm{ClN}_{2} \mathrm{O}_{4}:$ 677.40851, found: 677.41284 .

3ß-Hydroxy-30-(4-(3-bromobenzoyl)-1-piperazinyl)-olean-12-

ene-11,30-dione (4j). A white solid; yield, 90.9\%; mp 239.2$240.1{ }^{\circ} \mathrm{C} ;{ }^{1} \mathrm{H}$ NMR (400 MHz, chloroform- $d$ ) $\delta 7.57(\mathrm{~s}, 2 \mathrm{H}$, phenyl-H), 7.32 (q, $J=7.4 \mathrm{~Hz}, 2 \mathrm{H}$, phenyl-H), 5.68 (s, $1 \mathrm{H}, \mathrm{CH}-$ 12), 3.83-3.38 (m, 8H, piperazinyl-H), 3.22 (dd, $J=10.7$, $5.5 \mathrm{~Hz}, 1 \mathrm{H}, 3-\mathrm{OH}), 2.78(\mathrm{dt}, J=13.6,3.6 \mathrm{~Hz}, 1 \mathrm{H}, \mathrm{CH}-1), 2.33(\mathrm{~s}$, 1H, CH-9), 2.31-2.23 (m, 1H, CH-16), 1.36 (s, 3H, $\left.\mathrm{CH}_{3}-27\right), 1.24$ (s, 3H, $\left.\mathrm{CH}_{3}-25\right), 1.14$ (s, 3H, $\left.\mathrm{CH}_{3}-26\right), 1.12$ (s, 3H, $\left.\mathrm{CH}_{3}-29\right), 1.00$ (s, 3H, $\left.\mathrm{CH}_{3}-23\right), 0.82$ (s, 3H, $\left.\mathrm{CH}_{3}-24\right), 0.81$ (s, 3H, $\left.\mathrm{CH}_{3}-28\right), 0.70$ (d, $J=11.6 \mathrm{~Hz}, 1 \mathrm{H}, \mathrm{CH}-5) ;{ }^{13} \mathrm{C}$ NMR (101 MHz, chloroform- $d$ )

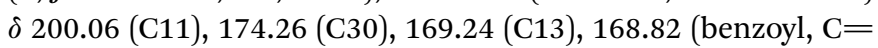
O), 137.05 (phenyl), 133.12 (phenyl), 130.25 (phenyl), 130.16 (phenyl), 128.59 (C12), 125.60 (phenyl), 122.79 (phenyl), 78.75 (C3), 61.79 (C9), 54.92 (C5), 48.11 (C18), 45.27 (C14), 43.92 (C20), 43.77 (C8), 43.26 (piperazinyl C), 39.11 (piperazinyl C), 37.68 (C4), 37.06 (C22), 33.15 (C10), 32.79 (C7), 31.78 (C17), 29.69 (C21), 28.39 (C29), 28.07 (C28), 27.28 (C23), 27.06 (C2), 26.68 (C15), 26.39 (C16), 23.15 (C27), 18.66 (C26), 17.46 (C6), 16.37 (C25), 15.56 (C24); HRMS (m/z): $[\mathrm{M}+\mathrm{Na}]^{+}$calcd for $\mathrm{C}_{41} \mathrm{H}_{57^{-}}$ $\mathrm{BrN}_{2} \mathrm{NaO}_{4}$ : 743.33994, found: 743.34621, 745.34582.

3ß-Hydroxy-30-(4-(4-bromobenzoyl)-1-piperazinyl)-olean-12ene-11,30-dione (4k). A white solid; yield, 91.1\%; $\mathrm{mp} 233.2-$ $235.4{ }^{\circ} \mathrm{C} ;{ }^{1} \mathrm{H}$ NMR $(400 \mathrm{MHz}$, chloroform- $d$ ) $\delta 7.57(\mathrm{~d}, J=8.1 \mathrm{~Hz}$, 2H, phenyl-H), 7.30 (d, $J=8.1 \mathrm{~Hz}, 2 \mathrm{H}$, phenyl-H), $5.67(\mathrm{~s}, 1 \mathrm{H}$, CH-12), 3.68 (s, 8H, piperazinyl-H), 3.22 (dd, $J=10.7,5.5 \mathrm{~Hz}$, 1H, 3-OH), 2.81-2.76 (m, 1H, CH-1), 2.33 (s, 1H, CH-9), 2.27 (d, J
$=11.7 \mathrm{~Hz}, 1 \mathrm{H}, \mathrm{CH}-16), 1.36\left(\mathrm{~s}, 3 \mathrm{H}, \mathrm{CH}_{3}-27\right), 1.23\left(\mathrm{~s}, 3 \mathrm{H}, \mathrm{CH}_{3}-25\right)$, 1.14 (s, 3H, $\left.\mathrm{CH}_{3}-26\right), 1.12$ (s, 3H, $\left.\mathrm{CH}_{3}-29\right), 1.00$ (s, 3H, $\mathrm{CH}_{3}-23$ ), 0.82 (s, 3H, $\left.\mathrm{CH}_{3}-24\right), 0.81$ (s, 3H, $\left.\mathrm{CH}_{3}-28\right), 0.70$ (d, $J=11.6 \mathrm{~Hz}$, 1H, CH-5); ${ }^{13} \mathrm{C}$ NMR (101 MHz, chloroform-d) $\delta 200.06$ (C11), 174.25 (C30), 169.54 (C13), 169.25 (benzoyl, $\mathrm{C}=\mathrm{O}), 133.89$ (phenyl), 131.88 (phenyl), 128.84 (phenyl), 128.58 (C12), 124.45 (phenyl), 78.75 (C3), 61.79 (C9), 54.92 (C5), 48.15 (C18), 45.27 (C14), 43.91 (C20), 43.74 (C8), 43.27 (piperazinyl C), 39.11 (piperazinyl C), 37.67 (C4), 37.06 (C22), 33.21 (C10), 32.79 (C7), 31.78 (C17), 29.70 (C21), 28.38 (C29), 28.07 (C28), 27.28 (C23), 27.05 (C2), 26.68 (C15), 26.39 (C16), 23.15 (C27), 18.66 (C26), 17.46 (C6), 16.37 (C25), 15.56 (C24); HRMS (m/z): $[\mathrm{M}+\mathrm{H}]^{+}$calcd for $\mathrm{C}_{41} \mathrm{H}_{58} \mathrm{BrN}_{2} \mathrm{O}_{4}$ : 721.35800, found: 721.36354, 723.36266.

3ß-Hydroxy-30-(4-(3-fluorobenzoyl)-1-piperazinyl)-olean-12-ene11,30-dione (4l). A white solid; yield, 92.1\%; $\operatorname{mp~} 231.5-232.9{ }^{\circ} \mathrm{C}$; ${ }^{1} \mathrm{H}$ NMR (400 MHz, chloroform- $d$ ) $\delta 7.41(\mathrm{td}, J=7.8,5.5 \mathrm{~Hz}, 1 \mathrm{H}$, phenyl-H), 7.16 (dd, $J=21.9,8.1 \mathrm{~Hz}, 3 \mathrm{H}$, phenyl-H), $5.68(\mathrm{~s}, 1 \mathrm{H}$, CH-12), 3.71-3.44 (m, 8H, piperazinyl-H), 3.22 (dd, $J=10.8$, $5.5 \mathrm{~Hz}, 1 \mathrm{H}, 3-\mathrm{OH}$ ), 2.79 (dd, $J=13.4,3.7 \mathrm{~Hz}, 1 \mathrm{H}, \mathrm{CH}-1), 2.33$ (s, $1 \mathrm{H}, \mathrm{CH}-9), 2.28$ (d, J=12.4 Hz, $1 \mathrm{H}, \mathrm{CH}-16), 1.36$ (s, 3H, $\mathrm{CH}_{3}-27$ ), 1.23 (s, 3H, $\left.\mathrm{CH}_{3}-25\right), 1.14$ (s, 3H, $\left.\mathrm{CH}_{3}-26\right), 1.12$ (s, 3H, $\mathrm{CH}_{3}-29$ ), 1.00 (s, 3H, $\left.\mathrm{CH}_{3}-23\right), 0.82$ (s, 3H, $\left.\mathrm{CH}_{3}-24\right), 0.81$ (s, 3H, $\left.\mathrm{CH}_{3}-28\right)$, 0.70 (d, $J=11.5 \mathrm{~Hz}, 1 \mathrm{H}, \mathrm{CH}-5) ;{ }^{13} \mathrm{C}$ NMR (101 MHz, chloroformd) $\delta 200.06$ (C11), 174.26 (C30), 169.25 (C13), 169.04 (benzoyl, $\mathrm{C}=\mathrm{O}), 163.78$ (C-F), 161.30 (C-F), 137.17 (phenyl), 137.10 (phenyl), 130.52 (phenyl), 130.44 (phenyl), 128.59 (C12), 122.74 (phenyl), 122.71 (phenyl), 117.22 (phenyl), 117.01 (phenyl), 114.56 (phenyl), 114.33 (phenyl), 78.74 (C3), 61.78 (C9), 54.92 (C5), 48.11 (C18), 45.27 (C14), 43.92 (C20), 43.77 (C8), 43.26 (piperazinyl C), 39.11 (piperazinyl C), 37.68 (C4), 37.06 (C22), 33.15 (C10), 32.79 (C7), 31.77 (C17), 29.69 (C21), 28.39 (C29), 28.07 (C28), 27.28 (C23), 27.04 (C2), 26.68 (C15), 26.38 (C16), 23.14 (C27), 18.66 (C26), 17.46 (C6), 16.37 (C25), 15.56 (C24); HRMS $(m / z):[\mathrm{M}+\mathrm{H}]^{+}$calcd $\mathrm{C}_{41} \mathrm{H}_{58} \mathrm{FN}_{2} \mathrm{O}_{4}: 661.43806$, found: 661.44479.

33-Hydroxy-30-(4-(2,4-difluorobenzoyl)-1-piperazinyl)-olean-12ene-11,30-dione (4m). A white solid; yield, 92.0\%; $\mathrm{mp} 229.7-$ $231.1{ }^{\circ} \mathrm{C} ;{ }^{1} \mathrm{H}$ NMR (400 MHz, chloroform- $d$ ) $\delta 7.43(\mathrm{td}, J=8.2$, $6.3 \mathrm{~Hz}, 1 \mathrm{H}$, phenyl-H), $6.98(\mathrm{td}, J=8.2,2.4 \mathrm{~Hz}, 1 \mathrm{H}$, phenyl-H), $6.87(\mathrm{td}, J=9.2,2.4 \mathrm{~Hz}, 1 \mathrm{H}$, phenyl-H), 5.68 (s, 1H, CH-12), 3.89-3.53 (m, 6H, piperazinyl-H), 3.34 (s, 2H, piperazinyl- $\mathrm{H})$, $3.22(\mathrm{dd}, J=10.7,5.5 \mathrm{~Hz}, 1 \mathrm{H}, 3-\mathrm{OH}), 2.78(\mathrm{dt}, J=13.5$, $3.6 \mathrm{~Hz}, 1 \mathrm{H}, \mathrm{CH}-1), 2.33$ (s, 1H, CH-9), 2.32-2.26 (m, 1H, CH-16), 1.36 (s, 3H, $\left.\mathrm{CH}_{3}-27\right), 1.24$ (s, 3H, $\left.\mathrm{CH}_{3}-25\right), 1.13$ (s, 3H, $\left.\mathrm{CH}_{3}-26\right)$, 1.12 (s, 3H, $\left.\mathrm{CH}_{3}-29\right), 1.00$ (s, 3H, $\left.\mathrm{CH}_{3}-23\right), 0.82$ (s, 3H, $\left.\mathrm{CH}_{3}-24\right)$, 0.81 (s, 3H, $\left.\mathrm{CH}_{3}-28\right), 0.70$ (d, $\left.J=11.4 \mathrm{~Hz}, 1 \mathrm{H}, \mathrm{CH}-5\right) ;{ }^{13} \mathrm{C} \mathrm{NMR}$ (101 MHz, chloroform-d) $\delta 200.05$ (C11), 174.22 (C30), 169.24 (C13), 165.13 (C-F), 165.01 (C-F), 164.52 (benzoyl, C=O), 162.62 (C-F), 162.50 (C-F), 159.77 (C-F), 159.65 (C-F), 157.28 (C-F), 157.16 (C-F), 130.89 (phenyl), 130.84 (phenyl), 130.79 (phenyl), 130.74 (phenyl), 128.60 (C12), 119.80 (phenyl), 119.76 (phenyl), 119.62 (phenyl), 119.58 (phenyl), 112.58 (phenyl), 112.54 (phenyl), 112.36 (phenyl), 112.33 (phenyl), 104.51 (phenyl), 104.26 (phenyl), 104.00 (phenyl), 78.73 (C3), 61.78 (C9), 54.92 (C5), 48.06 (C18), 47.12 (C14), 45.26 (C20), 43.92 (C8), 43.82, 43.26 (C8), 42.31 (piperazinyl C), 39.10 (C4), 37.68 (C22), 37.06 (C10), 33.09 (C7), 32.78 (C17), 31.76 (C21), 28.07 (C28), 27.27 
(C23), 27.05 (C2), 26.67 (C15), 26.37 (C16), 23.14 (C27), 18.65 (C26), 17.46 (C6), 16.36 (C25), 15.56 (C24); HRMS (m/z): [M + H $]^{+}$ calcd for $\mathrm{C}_{41} \mathrm{H}_{57} \mathrm{~F}_{2} \mathrm{~N}_{2} \mathrm{O}_{4}$ : 679.42864, found: 679.43500 .

3 $\beta$-Hydroxy-30-(4-(3,5-difluorobenzoyl)-1-piperazinyl)-olean-12ene-11,30-dione (4n). A white solid; yield, 92.1\%; mp 236.3236.8 ${ }^{\circ} \mathrm{C} ;{ }^{1} \mathrm{H}$ NMR (400 MHz, chloroform- $d$ ) $\delta$ 7.05-6.79 $(\mathrm{m}, 3 \mathrm{H}$, phenyl-H), 5.67 (d, $J=2.1 \mathrm{~Hz}, 1 \mathrm{H}, \mathrm{CH}-12), 3.84-3.52(\mathrm{~m}, 6 \mathrm{H}$, piperazinyl-H), $3.42(\mathrm{~s}, 2 \mathrm{H}$, piperazinyl-H), $3.22(\mathrm{dd}, J=10.7$, $5.4 \mathrm{~Hz}, 1 \mathrm{H}, 3-\mathrm{OH}), 2.78(\mathrm{dt}, J=13.5,3.1 \mathrm{~Hz}, 1 \mathrm{H}, \mathrm{CH}-1), 2.33(\mathrm{~s}$, $1 \mathrm{H}, \mathrm{CH}-9), 2.27$ (d, J=13.1 Hz, $1 \mathrm{H}, \mathrm{CH}-16), 1.35$ (s, 3H, $\mathrm{CH}_{3}-27$ ), 1.23 (s, 3H, $\left.\mathrm{CH}_{3}-25\right), 1.13$ (s, 3H, $\left.\mathrm{CH}_{3}-26\right), 1.12$ (s, 3H, $\left.\mathrm{CH}_{3}-29\right)$, 1.00 (s, 3H, $\mathrm{CH}_{3}-23$ ), 0.81 (s, 3H, $\left.\mathrm{CH}_{3}-24\right), 0.80$ (s, 3H, $\mathrm{CH}_{3}-28$ ), 0.70 (d, $J=11.5 \mathrm{~Hz}, 1 \mathrm{H}, \mathrm{CH}-5) ;{ }^{13} \mathrm{C}$ NMR (101 MHz, chloroformd) $\delta 200.05$ (C11), 174.28 (C30), 169.21 (C13), 167.80 (benzoyl, $\mathrm{C}=\mathrm{O}), 167.77$ (C-F), 167.74 (C-F), 164.23 (C-F), $164.11(\mathrm{C}-\mathrm{F})$, 161.72 (C-F), 161.60 (C-F), 138.21 (phenyl), 138.13 (phenyl), 138.04 (phenyl), 128.58 (C12), 110.53 (phenyl), 110.45 (phenyl), 110.34 (phenyl), 110.26 (phenyl), 105.82 (phenyl), 105.57 (phenyl), 105.32 (phenyl), 78.72 (C3), 61.78 (C9), 54.91 (C5), 48.10 (C18), 45.26 (C14), 45.21 (20), 43.92 (C8), 43.76 (piperazinyl C), 43.26 (C19), 39.10 (piperazinyl C), 37.66 (C22), 37.06 (C10), 33.12 (C7), 32.78 (C17), 31.77 (C21), 28.38 (C29), 28.07 (C28), 27.27 (C23), 27.02 (C2), 26.67 (C15), 26.37 (C16), 23.14 (C27), 18.66 (C26), 17.46 (C6), 16.36 (C25), 15.57 (C24); HRMS $(\mathrm{m} / \mathrm{z}):[\mathrm{M}+\mathrm{H}]^{+}$calcd for $\mathrm{C}_{41} \mathrm{H}_{57} \mathrm{~F}_{2} \mathrm{~N}_{2} \mathrm{O}_{4}$ : 679.42864, found: 679.43500 .

3ß-Hydroxy-30-(4-(3-(trifluoromethyl)benzoyl)-1-piperazinyl)olean-12-ene-11,30-dione (4o). A white solid; yield, 90.8\%; mp 258.5-259.7 ${ }^{\circ} \mathrm{C} ;{ }^{1} \mathrm{H}$ NMR (400 MHz, chloroform- $d$ ) $\delta 7.71(\mathrm{~d}, J=$ $10.3 \mathrm{~Hz}, 2 \mathrm{H}$, phenyl-H), 7.65-7.53 (m, 2H, phenyl-H), $5.68(\mathrm{~s}, 1 \mathrm{H}$, $\mathrm{CH}-12$ ), 3.70 (m, 6H, piperazinyl-H), 3.44 (s, 2H, piperazinyl- $\mathrm{H}$ ), 3.22 (dd, $J=10.8,5.5 \mathrm{~Hz}, 1 \mathrm{H}, 3-\mathrm{OH}), 2.83-2.73$ (m, 1H, CH-1), 2.33 (s, 1H, CH-9), 2.28 (d, $J=11.4 \mathrm{~Hz}, 1 \mathrm{H}, \mathrm{CH}-16), 1.36$ (s, $\left.3 \mathrm{H}, \mathrm{CH}_{3}-27\right), 1.24$ (s, 3H, $\left.\mathrm{CH}_{3}-25\right), 1.14$ (s, 3H, $\left.\mathrm{CH}_{3}-26\right), 1.12$ (s, $\left.3 \mathrm{H}, \mathrm{CH}_{3}-29\right), 0.98$ (s, 3H, $\left.\mathrm{CH}_{3}-23\right), 0.82$ (s, 3H, $\left.\mathrm{CH}_{3}-24\right), 0.81$ (s, $\left.3 \mathrm{H}, \mathrm{CH}_{3}-28\right), 0.70$ (d, $\left.J=11.6 \mathrm{~Hz}, 1 \mathrm{H}\right) ;{ }^{13} \mathrm{C} \mathrm{NMR}(101 \mathrm{MHz}$, chloroform- $d$ ) $\delta 200.06$ (C11), 174.27 (C30), 169.23 (C13), 168.94 (benzoyl, $\mathrm{C}=\mathrm{O}$ ), 138.91 (phenyl), 131.09 (phenyl), 130.38 (phenyl), 129.27 ( $\left.\mathrm{CF}_{3}\right), 128.59$ (C12), 126.84 (phenyl), 124.21 (phenyl), 124.17 (phenyl), 78.74 (C3), 61.79 (C9), 54.92 (C5), 48.12 (C18), 45.27 (C14), 43.93 (C20), 43.76 (C8), 43.26 (piperazinyl C), 39.11 (piperazinyl C), 37.67 (C4), 37.06 (C22), 33.15 (C10), 32.79 (C7), 31.78 (C17), 29.70 (C21), 28.39 (C29), 28.07 (C28), 27.28 (C23), 27.04 (C2), 26.67 (C15), 26.38 (C16), 23.14 (C27), 18.66 (C26), 17.46 (C6), 16.37 (C25), 15.56 (C24); HRMS $(\mathrm{m} / \mathrm{z}):[\mathrm{M}+\mathrm{H}]^{+}$calcd for $\mathrm{C}_{42} \mathrm{H}_{58} \mathrm{~F}_{3} \mathrm{~N}_{2} \mathrm{O}_{4}$ : 711.43487, found: 711.44181.

3 $\beta$-Hydroxy-30-(4-(4-(trifluoromethyl)benzoyl)-1-piperazinyl)olean-12-ene-11,30-dione (4p). A white solid; yield, 90.8\%; mp 237.2-239.2 ${ }^{\circ} \mathrm{C} ;{ }^{1} \mathrm{H}$ NMR (400 MHz, chloroform- $d$ ) $\delta 7.72(\mathrm{~d}, J=$ $8.0 \mathrm{~Hz}, 2 \mathrm{H}$, phenyl-H), 7.55 (d, $J=8.0 \mathrm{~Hz}, 2 \mathrm{H}$, phenyl-H), 5.68 (s, $1 \mathrm{H}, \mathrm{CH}-12), 3.69$ (m, 6H, piperazinyl-H), 3.41 (s, 2H, piperazinyl$\mathrm{H}), 3.23(\mathrm{dd}, J=10.7,5.6 \mathrm{~Hz}, 1 \mathrm{H}, 3-\mathrm{OH}), 2.84-2.75(\mathrm{~m}, 1 \mathrm{H}, \mathrm{CH}-$ 1), $2.34(\mathrm{~s}, 1 \mathrm{H}, \mathrm{CH}-9), 2.30-2.21(\mathrm{~m}, 1 \mathrm{H}, \mathrm{CH}-16), 1.36(\mathrm{~s}, 3 \mathrm{H}$, $\left.\mathrm{CH}_{3}-27\right), 1.24$ (s, 3H, $\left.\mathrm{CH}_{3}-25\right), 1.14\left(\mathrm{~s}, 3 \mathrm{H}, \mathrm{CH}_{3}-26\right), 1.13$ (s, 3H, $\left.\mathrm{CH}_{3}-29\right), 1.01$ (s, 3H, $\left.\mathrm{CH}_{3}-23\right), 0.83$ (s, 3H, $\left.\mathrm{CH}_{3}-24\right), 0.81$ (s, 3H, $\left.\mathrm{CH}_{3}-28\right), 0.70$ (d, $\left.J=11.6 \mathrm{~Hz}, 1 \mathrm{H}, \mathrm{CH}-5\right) ;{ }^{13} \mathrm{C}$ NMR (101 MHz, chloroform- $d$ ) $\delta 200.07$ (C11), 174.28 (C30), 169.26 (C13), 169.05 (benzoyl, $\mathrm{C}=\mathrm{O}$ ), 138.65 (phenyl), 132.15 (phenyl), 131.82 (phenyl), 130.39 (phenyl), 128.57 (C12), 127.48 (phenyl), 125.82 $\left(\mathrm{CF}_{3}\right), 125.78\left(\mathrm{CF}_{3}\right), 125.74\left(\mathrm{CF}_{3}\right), 125.70\left(\mathrm{CF}_{3}\right), 124.94$ (phenyl), 122.23 (phenyl), 78.74 (C3), 61.79 (C9), 54.92 (C5), 48.18 (C18), 45.28 (C14), 43.92 (C20), 43.70 (C8), 43.27 (piperazinyl C), 39.10 (piperazinyl C), 37.66 (C4), 37.06 (C22), 33.24 (C10), 32.78 (C7), 31.79 (C17), 29.69 (C21), 28.38 (C29), 28.07 (C28), 27.27 (C23), 27.04 (C2), 26.67 (C15), 26.39 (C16), 23.14 (C27), 18.66 (C26), 17.46 (C6), 16.37 (C25), 15.56 (C24); $\operatorname{HRMS}(\mathrm{m} / \mathrm{z}):[\mathrm{M}+\mathrm{Na}]^{+}$calcd for $\mathrm{C}_{42} \mathrm{H}_{57} \mathrm{~F}_{3} \mathrm{~N}_{2} \mathrm{NaO}_{4}$ : 733.41681, found: 733.42400 .

33-Hydroxy-30-(4-(4-cyanobenzoyl)-1-piperazinyl)-olean-12-ene11,30-dione (4q). A white solid; yield, 86.4\%; mp $235.5-237.7^{\circ} \mathrm{C}$; ${ }^{1} \mathrm{H}$ NMR (400 MHz, chloroform-d) $\delta 7.75(\mathrm{~d}, J=8.2 \mathrm{~Hz}, 2 \mathrm{H}$, phenyl-H), 7.57-7.50 (m, 2H, phenyl-H), 5.66 (s, 1H, CH-12), 3.84-3.35 (m, 8H, piperazinyl-H), 3.22 (dd, $J=10.7,5.5 \mathrm{~Hz}$, $1 \mathrm{H}, 3-\mathrm{OH}), 2.78$ (dt, $J=13.5,3.5 \mathrm{~Hz}, 1 \mathrm{H}, \mathrm{CH}-1), 2.33(\mathrm{~s}, 1 \mathrm{H}, \mathrm{CH}-$ 9), 2.28-2.20 (m, 1H, CH-16), $1.36\left(\mathrm{~s}, 3 \mathrm{H}, \mathrm{CH}_{3}-27\right), 1.23(\mathrm{~s}, 3 \mathrm{H}$, $\left.\mathrm{CH}_{3}-25\right), 1.14$ (s, 3H, $\left.\mathrm{CH}_{3}-26\right), 1.12$ (s, 3H, $\left.\mathrm{CH}_{3}-29\right), 1.00$ (s, 3H, $\left.\mathrm{CH}_{3}-23\right), 0.82$ (s, 3H, $\left.\mathrm{CH}_{3}-24\right), 0.81$ (s, 3H, $\left.\mathrm{CH}_{3}-28\right), 0.70$ (d, $J=$ $11.6 \mathrm{~Hz}, 1 \mathrm{H}, \mathrm{CH}-5) ;{ }^{13} \mathrm{C}$ NMR (101 MHz, chloroform-d) $\delta 200.09$ (C11), 174.30 (C30), 169.28 (C13), 168.45 (benzoyl, C=O), 139.42 (phenyl), 132.56 (phenyl), 128.55 (C12), 127.82 (phenyl), 117.92 (phenyl), 113.89 (CN), 78.72 (C3), 61.80 (C9), 54.92 (C5), 48.21 (C18), 45.28 (C14), 43.93 (C20), 43.66 (C8), 43.27 (piperazinyl C), 39.11 (piperazinyl C), 37.64 (C4), 37.07 (C22), 33.29 (C10), 32.78 (C7), 31.79 (C17), 29.69 (C21), 28.38 (C29), 28.07 (C28), 27.27 (C23), 27.03 (C2), 26.67 (C15), 26.39 (C16), 23.14 (C27), 18.66 (C26), 17.45 (C6), 16.37 (C25), 15.56 (C24); $\operatorname{HRMS~(m/z):~[M+~H~}]^{+}$ calcd for $\mathrm{C}_{42} \mathrm{H}_{58} \mathrm{~N}_{3} \mathrm{O}_{4}$ : 668.44273, found: 668.44912.

3 $\beta$-Hydroxy-30-(4-cyclohexanecarbonyl-1-piperazinyl)-olean-12ene-11,30-dione (4r). A white solid; yield, 86.4\%; mp 213.6$215.4{ }^{\circ} \mathrm{C}$; ${ }^{1} \mathrm{H}$ NMR (400 MHz, chloroform- $d$ ) $\delta 5.68$ (s, $1 \mathrm{H}, \mathrm{CH}-$ 12), 3.69-3.46 (m, 8H, piperazinyl-H), 3.22 (dd, $J=10.7$, $5.5 \mathrm{~Hz}, 1 \mathrm{H}, 3-\mathrm{OH}), 2.78$ (dt, $J=13.4,3.5 \mathrm{~Hz}, 1 \mathrm{H}, \mathrm{CH}-1), 2.46(\mathrm{tt}, J$ $=11.6,3.4 \mathrm{~Hz}, 1 \mathrm{H}$, cyclohexanecarbonyl-CH), 2.34 (s, 1H, CH-9), 2.29 (dd, $J=12.7,3.5 \mathrm{~Hz}, 1 \mathrm{H}, \mathrm{CH}-16), 1.36$ (s, 3H, $\left.\mathrm{CH}_{3}-27\right), 1.30-$ 1.25 (m, 10H, cyclohexane), $1.23\left(\mathrm{~s}, 3 \mathrm{H}, \mathrm{CH}_{3}-25\right), 1.14(\mathrm{~s}, 3 \mathrm{H}$, $\left.\mathrm{CH}_{3}-26\right), 1.12$ (s, 3H, $\left.\mathrm{CH}_{3}-29\right), 1.01$ (s, 3H, $\left.\mathrm{CH}_{3}-23\right), 0.82$ (s, 3H, $\left.\mathrm{CH}_{3}-24\right), 0.81$ (s, 3H, $\left.\mathrm{CH}_{3}-28\right), 0.70$ (d, $\left.=11.5 \mathrm{~Hz}, 1 \mathrm{H}, \mathrm{CH}-5\right) ;{ }^{13} \mathrm{C}$ NMR (101 MHz, chloroform-d) $\delta 200.05$ (C11), 174.82 (cyclohexanecarbonyl, $\mathrm{C}=\mathrm{O}$ ), 174.20 (C30), 169.29 (C13), 128.58 (C12), 78.74 (C3), 61.77 (C9), 54.92 (C5), 48.07 (C18), 45.33 (C14), 45.26 (C20), 43.91 (C8), 43.82 (piperazinyl C), 43.26 (cyclohexane), 41.60 (C19), 40.39 (piperazinyl C), 39.11 (C4), 37.70 (C22), 37.06 (C10), 33.08 (C7), 32.79 (C17), 31.76 (C21), 29.69 (cyclohexane), 29.36 (cyclohexane), 29.29 (C29), 28.39 (C28), 28.07 (C23), 27.28 (C2), 27.03 (C15), 26.69 (C16), 26.38 (cyclohexane), 25.79 (cyclohexane), 25.76 (cyclohexane), 23.15 (C27), 18.66 (C26), 17.46 (C6), 16.36 (C25), 15.56 (C24); HRMS (m/z): [M $+\mathrm{H}]^{+}$calcd for $\mathrm{C}_{41} \mathrm{H}_{65} \mathrm{~N}_{2} \mathrm{O}_{4}$ : 649.49443, found: 649.50050 .

3 $\beta$-Hydroxy-30-(4-cyclopropanecarbonyl-1-piperazinyl)-olean12-ene-11,30-dione (4s). A white solid; yield, 84.8\%; mp 210.7$212.2{ }^{\circ} \mathrm{C} ;{ }^{1} \mathrm{H}$ NMR $(400 \mathrm{MHz}$, chloroform- $d$ ) $\delta 5.70(\mathrm{~d}, J=5.2 \mathrm{~Hz}$, 1H, CH-12), 3.67 (m, 8H, piperazinyl-H), 3.23 (dd, $J=11.0$, $5.4 \mathrm{~Hz}, 1 \mathrm{H}, 3-\mathrm{OH}), 2.79$ (d, J=13.3 Hz, 1H, CH-1), $2.32(\mathrm{~m}, 2 \mathrm{H}$, $\mathrm{CH}-9 / 16), 1.37$ (s, 3H, $\left.\mathrm{CH}_{3}-27\right), 1.24$ (s, 3H, $\left.\mathrm{CH}_{3}-25\right), 1.14$ (s, 3H, 
$\left.\mathrm{CH}_{3}-26\right), 1.12$ (s, 3H, $\left.\mathrm{CH}_{3}-29\right), 1.01$ (s, 3H, $\left.\mathrm{CH}_{3}-23\right), 0.82$ (s, 3H, $\left.\mathrm{CH}_{3}-24\right), 0.81$ (s, 3H, $\left.\mathrm{CH}_{3}-28\right), 0.70$ (d, $\left.J=11.6 \mathrm{~Hz}, 1 \mathrm{H}, \mathrm{CH}-5\right) ;{ }^{13} \mathrm{C}$ NMR (101 MHz, chloroform- $d$ ) $\delta 200.06$ (C11), 174.24 (C30), 172.34 (cyclopropanecarbonyl, $\mathrm{C}=\mathrm{O}$ ), 169.31 (C13), 128.59 (C12), 78.73 (C3), 61.78 (C9), 54.92 (C5), 48.07 (C18), 45.26 (C14), 43.93 (C20), 43.84 (C8), 43.26 (piperazinyl C), 42.09 (C19), 39.10 (piperazinyl C), 37.71 (C22), 37.06 (C10), 33.07 (C7), 32.79 (C17), 31.77 (C21), 29.69 (C29), 28.40 (C28), 28.07 (C23), 27.28 (C2), 27.04 (C15), 26.69 (C16), 23.15 (C27), 18.66 (C26), 17.46 (C6), 16.37 (C25), 15.57 (C24), 10.97 (cyclopropane), 7.72 (cyclopropane), 7.70 (cyclopropane); HRMS $(\mathrm{m} / \mathrm{z}):[\mathrm{M}+\mathrm{Na}]^{+}$calcd for $\mathrm{C}_{38} \mathrm{H}_{58} \mathrm{~N}_{2} \mathrm{NaO}_{4}$ : 629.42943, found: 629.43642 .

3 $\beta$-Hydroxy-30-(4-(6-chloronicotinoyl)-1-piperazinyl)-olean-12ene-11,30-dione (4t). A white solid; yield, 94.6\%; $\mathrm{mp} 244.5-$ $246.3{ }^{\circ} \mathrm{C} ;{ }^{1} \mathrm{H}$ NMR $(400 \mathrm{MHz}$, chloroform- $d$ ) $\delta 8.47(\mathrm{~d}, J=2.3 \mathrm{~Hz}$, $1 \mathrm{H}$, pyridyl-H), 7.76 (dd, $J=8.2,2.4 \mathrm{~Hz}, 1 \mathrm{H}$, pyridyl-H), $7.43(\mathrm{~d}, J$ $=8.1 \mathrm{~Hz}, 1 \mathrm{H}$, pyridyl-H), $5.67(\mathrm{~s}, 1 \mathrm{H}, \mathrm{CH}-12), 3.71-3.47(\mathrm{~m}, 8 \mathrm{H}$, piperazinyl-H), 3.22 (dd, $J=10.7,5.5 \mathrm{~Hz}, 1 \mathrm{H}, 3-\mathrm{OH}), 2.78(\mathrm{dt}, J=$ 13.3, 3.5 Hz, 1H, CH-1), 2.33 (s, 1H, CH-9), 2.30-2.21 (m, $1 \mathrm{H}$, $\mathrm{CH}-16), 1.36$ (s, 3H, $\left.\mathrm{CH}_{3}-27\right), 1.24$ (s, 3H, $\left.\mathrm{CH}_{3}-25\right), 1.14$ (s, 3H, $\left.\mathrm{CH}_{3}-26\right), 1.12$ (s, 3H, $\left.\mathrm{CH}_{3}-29\right), 1.00$ (s, 3H, $\left.\mathrm{CH}_{3}-23\right), 0.82$ (s, 3H, $\left.\mathrm{CH}_{3}-24\right), 0.81$ (s, 3H, $\left.\mathrm{CH}_{3}-28\right), 0.70$ (d, $\left.J=11.6 \mathrm{~Hz}, 1 \mathrm{H}, \mathrm{CH}-5\right) ;{ }^{13} \mathrm{C}$ NMR (101 MHz, chloroform- $d$ ) $\delta 200.05$ (C11), 174.30 (C30), 169.22 (C13), 166.88 (chloronicotinoyl, $\mathrm{C}=\mathrm{O}), 153.02$ (pyridyl), 148.12 (pyridyl), 138.01 (pyridyl), 129.72 (pyridyl), 128.57 (C12), 124.51 (pyridyl), 78.74 (C3), 61.79 (C9), 54.92 (C5), 48.16 (C18), 45.27 (C14), 43.93 (C20), 43.72 (C8), 43.27 (piperazinyl C), 39.13 (piperazinyl C), 39.11 (C4), 37.65 (C22), 37.06 (C10), 33.20 (C7), 32.78 (C17), 31.79 (C21), 28.38 (C29), 28.07 (C28), 27.28 (C23), 27.04 (C2), 26.67 (C15), 26.39 (C16), 23.15 (C27), 18.66 (C26), 17.46 (C6), 16.37 (C25), 15.56 (C24); HRMS (m/z): [M + H $]^{+}$calcd for $\mathrm{C}_{40} \mathrm{H}_{57} \mathrm{ClN}_{3} \mathrm{O}_{4}$ : 678.40376, found: 678.41033 .

3 $\beta$-Hydroxy-30-(4-(2-(thiophen-2-yl)acetyl)-1-piperazinyl)-olean12-ene-11,30-dione (4u). A white solid; yield, 87.5\%; mp 218.5$220.2{ }^{\circ} \mathrm{C} ;{ }^{1} \mathrm{H}$ NMR $(400 \mathrm{MHz}$, chloroform- $d$ ) $\delta 7.22$ (dd, $J=5.1$, $1.2 \mathrm{~Hz}, 1 \mathrm{H}$, thiophen-2-yl-H), 6.97 (dd, $J=5.2,3.5 \mathrm{~Hz}, 1 \mathrm{H}$, thiophen-2-yl-H), 6.91 (d, $J=3.4 \mathrm{~Hz}, 1 \mathrm{H}$, thiophen-2-yl-H), 5.67 (s, 1H, CH-12), 3.94 [s, 2H, (thiophen-2-yl)acetyl- $\left.\mathrm{CH}_{2}\right], 3.56(\mathrm{~m}$, $8 \mathrm{H}$, piperazinyl-H), 3.23 (dd, $J=10.7,5.6 \mathrm{~Hz}, 1 \mathrm{H}, 3-\mathrm{OH}), 2.79$ (dt, $J=13.5,3.5 \mathrm{~Hz}, 1 \mathrm{H}, \mathrm{CH}-1), 2.33$ (s, 1H, CH-9), 2.31-2.23 (m, 1H, CH-16), 1.36 (s, 3H, $\left.\mathrm{CH}_{3}-27\right), 1.21$ (s, 3H, $\left.\mathrm{CH}_{3}-25\right), 1.13$ (s, $\left.3 \mathrm{H}, \mathrm{CH}_{3}-26\right), 1.12$ (s, 3H, $\left.\mathrm{CH}_{3}-29\right), 1.00$ (s, 3H, $\left.\mathrm{CH}_{3}-23\right), 0.82$ (s, $\left.3 \mathrm{H}, \mathrm{CH}_{3}-24\right), 0.81$ (s, 3H, $\left.\mathrm{CH}_{3}-28\right), 0.70$ (d, $J=11.8 \mathrm{~Hz}, 1 \mathrm{H}, \mathrm{CH}-$ 5); ${ }^{13} \mathrm{C}$ NMR (101 MHz, chloroform-d) $\delta 200.05$ (C11), 174.23 (C30), 169.25 (C13), 168.62 [(thiophen-2-yl)acetyl, C=O], 135.98 (thiophen-2-yl), 128.58 (C12), 126.98 (thiophen-2-yl), 126.13 (thiophen-2-yl), 124.94 (thiophen-2-yl), 78.74 (C3), 61.77 (C9), 54.91 (C5), 48.02 (C18), 46.16 (C14), 45.26 (C20), 43.90 (C8), 43.82 (piperazinyl C), 43.25 (C19), 41.98 (piperazinyl C), 39.11 (C4), 37.68 (C22), 37.05 (C10), 35.17 [(thiophen-2-yl)acetyl- $\mathrm{CH}_{2}$ ], 33.00 (C7), 32.79 (C17), 31.75 (C21), 29.69 (C29), 28.38 (C28), 28.07 (C23), 27.28 (C2), 27.03 (C15), 26.67 (C16), 23.14 (C27), 18.65 (C26), 17.46 (C6), 16.37 (C25), 15.57 (C24); HRMS (m/z): [M $+\mathrm{Na}]^{+}$calcd for $\mathrm{C}_{40} \mathrm{H}_{58} \mathrm{~N}_{2} \mathrm{NaO}_{4} \mathrm{~S}: 685.40150$, found: 685.40759 .

3 $\beta$-Hydroxy-30-(4-(2-(4-fluorophenyl)acetyl)-1-piperazinyl)olean-12-ene-11,30-dione (4v). A white solid; yield, 91.3\%; mp 212.7-213.3 ${ }^{\circ} \mathrm{C} ;{ }^{1} \mathrm{H}$ NMR (400 MHz, chloroform- $d$ ) $\delta 7.21$ (dd, $J=$
8.3, $5.2 \mathrm{~Hz}, 2 \mathrm{H}$, phenyl), $7.03(\mathrm{t}, J=8.3 \mathrm{~Hz}, 2 \mathrm{H}$, phenyl), 5.66 (s, $1 \mathrm{H}, \mathrm{CH}-12$ ), 3.72 [s, 2H, (4-fluorophenyl)acetyl- $\mathrm{CH}_{2}$ ], $3.53(\mathrm{~m}$, $8 \mathrm{H}$, piperazinyl-H), 3.22 (dd, $J=10.7,5.4 \mathrm{~Hz}, 1 \mathrm{H}, 3-\mathrm{OH}), 2.79$ (d, $J=13.3 \mathrm{~Hz}, 1 \mathrm{H}, \mathrm{CH}-1), 2.33$ (s, 1H, CH-9), 2.26 (d, $J=12.4 \mathrm{~Hz}$, 1H, CH-16), 1.36 (s, 3H, $\left.\mathrm{CH}_{3}-27\right), 1.20$ (s, 3H, $\left.\mathrm{CH}_{3}-25\right), 1.14$ (s, $\left.3 \mathrm{H}, \mathrm{CH}_{3}-26\right), 1.12$ (s, 3H, $\left.\mathrm{CH}_{3}-29\right), 1.00$ (s, 3H, $\left.\mathrm{CH}_{3}-23\right), 0.81$ (s, $\left.6 \mathrm{H}, \mathrm{CH}_{3}-24 / 28\right), 0.70$ (d, $\left.J=11.5 \mathrm{~Hz}, 1 \mathrm{H}, \mathrm{CH}-5\right) ;{ }^{13} \mathrm{C}$ NMR (101 MHz, chloroform- $d$ ) $\delta 200.06$ (C11), 174.24 (C30), 169.53 (C13), 169.27 [(4-fluorophenyl)acetyl, C=O], 163.04 (C-F), 160.60 (CF), 130.26 (phenyl), 130.22 (phenyl), 130.14 (phenyl), 128.56 (C12), 115.81 (phenyl), 115.60 (phenyl), 78.73 (C3), 61.77 (C9), 54.91 (C5), 48.05 (C18), 45.94 (C14), 45.26 [(4-fluorophenyl) acetyl, $\mathrm{CH}_{2}$ ], 43.89 (C20), 43.79 (C8), 43.25 (piperazinyl C), 41.86 (C19), 39.90 (piperazinyl C), 39.10 (C1), 37.67 (C4), 37.05 (C22), 33.03 (C10), 32.78 (C7), 31.75 (C17), 29.69 (C21), 28.37 (C29), 28.07 (C28), 27.27 (C23), 27.02 (C2), 26.67 (C15), 26.37 (C16), 23.14 (C27), 18.65 (C26), 17.46 (C6), 16.37 (C25), 15.57 (C24); HRMS (m/z): $[\mathrm{M}+\mathrm{H}]^{+}$calcd for $\mathrm{C}_{42} \mathrm{H}_{60} \mathrm{FN}_{2} \mathrm{O}_{4}$ : 675.45371, found: 675.46080 .

3 $\beta$-Hydroxy-30-(4-(2-(4-chlorophenyl)acetyl)-1-piperazinyl)-

olean-12-ene-11,30-dione ( $\mathbf{4 w}$ ). A white solid; yield, 90.0\%; mp 239.7-240.5 ${ }^{\circ} \mathrm{C} ;{ }^{1} \mathrm{H}$ NMR (400 MHz, chloroform- $d$ ) $\delta 7.31(\mathrm{~d}, J=$ $8.3 \mathrm{~Hz}, 2 \mathrm{H}$, phenyl), 7.18 (d, $J=8.1 \mathrm{~Hz}, 2 \mathrm{H}$, phenyl), 5.66 (s, 1H, $\mathrm{CH}-12), 3.71$ [s, 2H, (4-chlorophenyl)acetyl- $\mathrm{CH}_{2}$ ], 3.65-3.39 (m, $8 \mathrm{H}$, piperazinyl-H), 3.22 (dd, $J=10.7,5.5 \mathrm{~Hz}, 1 \mathrm{H}, 3-\mathrm{OH}), 2.78$ $(\mathrm{dt}, J=13.5,3.6 \mathrm{~Hz}, 1 \mathrm{H}, \mathrm{CH}-1), 2.33$ (s, 1H, CH-9), 2.30-2.22 (m, 1H, CH-16), 1.36 (s, 3H, $\mathrm{CH}_{3}-27$ ), 1.20 (s, 3H, $\left.\mathrm{CH}_{3}-25\right), 1.13$ (s, $\left.3 \mathrm{H}, \mathrm{CH}_{3}-26\right), 1.12$ (s, 3H, $\left.\mathrm{CH}_{3}-29\right), 1.00$ (s, 3H, $\left.\mathrm{CH}_{3}-23\right), 0.80$ (s, $\left.6 \mathrm{H}, \mathrm{CH}_{3}-24 / 28\right), 0.70$ (d, $\left.J=11.8 \mathrm{~Hz}, 1 \mathrm{H}, \mathrm{CH}-5\right) ;{ }^{13} \mathrm{C}$ NMR (101 MHz, chloroform-d) $\delta 200.05$ (C11), 174.24 (C30), 169.26 (C13), 169.22 [(4-chlorophenyl)acetyl, $\mathrm{C}=\mathrm{O}$ ], 133.01 (phenyl), 132.92 (phenyl), 130.01 (phenyl), 128.96 (phenyl), 128.57 (C12), 78.74 (C3), 61.78 (C9), 54.92 (C5), 48.05 (C18), 45.93 (C14), 45.26 [(4chlorophenyl)acetyl, $\mathrm{CH}_{2}$ ], 43.89 (C20), 43.79 (C8), 43.25 (piperazinyl C), 41.86 (C19), 40.07 (piperazinyl C), 39.10 (C1), 37.67 (C4), 37.06 (C22), 33.03 (C1), 32.79 (C7), 31.75 (C17), 29.69 (C21), 28.37 (C29), 28.07 (C28), 27.28 (C23), 27.02 (C2), 26.67 (C15), 26.37 (C16), 23.14 (C27), 18.65 (C26), 17.46 (C6), 16.37 (C25), 15.56 (C24); HRMS $(\mathrm{m} / \mathrm{z}):[\mathrm{M}+\mathrm{H}]^{+}$calcd for $\mathrm{C}_{42} \mathrm{H}_{60} \mathrm{ClN}_{2} \mathrm{O}_{4}$ : 691.42416, found: 691.43121 .

3 $\beta$-Hydroxy-30-(4-(morpholine-4-carbonyl)-1-piperazinyl)-olean12-ene-11,30-dione (4x). A white solid; yield, 88.2\%; mp 211.7$213.7{ }^{\circ} \mathrm{C} ;{ }^{1} \mathrm{H}$ NMR (400 MHz, chloroform- $d$ ) $\delta 5.68(\mathrm{~s}, 1 \mathrm{H}, \mathrm{CH}-$ 12), 3.80-3.53 (m, 8H, piperazinyl- $\mathrm{H}), 3.26(\mathrm{~m}, 9 \mathrm{H}$, morpholinyl-H/3-OH), 2.78 (dt, $J=13.4,3.4 \mathrm{~Hz}, 1 \mathrm{H}, \mathrm{CH}-1), 2.34$ (s, 1H, CH-9), 2.28 (d, J=12.9 Hz, 1H, CH-16), 1.36 (s, 3H, $\mathrm{CH}_{3}-$ 27), 1.22 (s, 3H, $\left.\mathrm{CH}_{3}-25\right), 1.14$ (s, 3H, $\left.\mathrm{CH}_{3}-26\right), 1.12$ (s, 3H, $\mathrm{CH}_{3}$ 29), 1.01 (s, 3H, $\mathrm{CH}_{3}-23$ ), 0.82 (s, 3H, $\left.\mathrm{CH}_{3}-24\right), 0.81$ (s, 3H, $\mathrm{CH}_{3}-$ 28), 0.70 (d, $J=11.5 \mathrm{~Hz}, 1 \mathrm{H}, \mathrm{CH}-5) ;{ }^{13} \mathrm{C}$ NMR (101 MHz, chloroform- $d$ ) $\delta 200.10$ (C11), 174.13 (C30), 169.41 (C13), 163.56 (morpholine-4-carbonyl, $\mathrm{C}=\mathrm{O}$ ), 128.54 (C12), 78.72 (C3), 61.78 (C9), 54.91 (C5), 48.13 (C18), 47.14 (morpholinyl-C), 46.91 (morpholinyl-C), 45.26 (C14), 43.88 (C20), 43.77 (C8), 43.26 (piperazinyl C), 39.11 (piperazinyl C), 37.69 (C4), 37.06 (C22), 33.20 (C10), 32.78 (C7), 31.76 (C17), 29.68 (C21), 28.40 (C29), 28.07 (C28), 27.27 (C23), 27.02 (C2), 26.69 (C15), 26.39 (C16), 23.14 (C27), 18.65 (C26), 17.46 (C6), 16.37 (C25), 15.57 (C24); 
HRMS $(\mathrm{m} / \mathrm{z}):[\mathrm{M}+\mathrm{H}]^{+}$calcd $\mathrm{C}_{39} \mathrm{H}_{62} \mathrm{~N}_{3} \mathrm{O}_{5}$ : 652.46895, found: 652.47377.

$\beta$-Hydroxy-30-(4-(2-methoxyacetyl)-1-piperazinyl)-olean-12-ene11,30-dione (4y). A white solid; yield, 85.4\%; $\mathrm{mp} 221.7-222.5^{\circ} \mathrm{C}$; ${ }^{1} \mathrm{H}$ NMR $(400 \mathrm{MHz}$, chloroform- $d) \delta 5.67(\mathrm{~d}, J=3.8 \mathrm{~Hz}, 1 \mathrm{H}, \mathrm{CH}-$ 12), $4.12\left(\mathrm{~s}, 2 \mathrm{H}\right.$, methoxyacetyl- $\left.\mathrm{CH}_{2}\right), 3.57$ (d, $J=49.5 \mathrm{~Hz}, 8 \mathrm{H}$, piperazinyl-H), $3.43\left(\mathrm{~d}, J=3.6 \mathrm{~Hz}, 3 \mathrm{H}\right.$, methoxyacetyl- $\left.\mathrm{CH}_{3}\right), 3.22$ $(\mathrm{dt}, J=10.4,4.6 \mathrm{~Hz}, 1 \mathrm{H}, 3-\mathrm{OH}), 2.77\left(\mathrm{dt}, J=13.4,3.8 \mathrm{~Hz}, 1 \mathrm{H}, \mathrm{CH}^{-}\right.$ 1), $2.33(\mathrm{~d}, J=3.7 \mathrm{~Hz}, 1 \mathrm{H}, \mathrm{CH}-9), 2.28(\mathrm{~d}, J=13.1 \mathrm{~Hz}, 1 \mathrm{H}, \mathrm{CH}-$ 16), 1.35 (s, 3H, $\left.\mathrm{CH}_{3}-27\right), 1.22\left(\mathrm{~s}, 3 \mathrm{H}, \mathrm{CH}_{3}-25\right), 1.12\left(\mathrm{~s}, 3 \mathrm{H}, \mathrm{CH}_{3^{-}}\right.$ 26), 1.11 (s, 3H, $\left.\mathrm{CH}_{3}-29\right), 0.99$ (s, 3H, $\left.\mathrm{CH}_{3}-23\right), 0.81$ (s, 3H, $\mathrm{CH}_{3}$ 24), 0.80 (s, 3H, $\left.\mathrm{CH}_{3}-28\right), 0.69$ (d, $\left.J=12.0 \mathrm{~Hz}, 1 \mathrm{H}, \mathrm{CH}-5\right) ;{ }^{13} \mathrm{C}$ NMR (101 MHz, chloroform- $d$ ) $\delta 200.05$ (C11), 174.24 (C30), 169.27 (C13), 167.80 (methoxyacetyl, $\mathrm{C}=\mathrm{O}), 128.58$ (C12), 78.71 (C3), 71.96 (methoxyacetyl, $\mathrm{CH}_{2}$ ), 61.77 (C9), 59.12 (methoxyacetyl, $\mathrm{CH}_{3}$ ), 54.91 (C5), 48.06 (C18), 45.26 (C14), 43.90 (C20), 43.82 (C8), 43.26 (piperazinyl C), 39.10 (piperazinyl C), 37.69 (C4), 37.05 (C22), 33.07 (C10), 32.78 (C7), 31.76 (C17), 29.68 (C21), 28.39 (C29), 28.07 (C28), 27.27 (C23), 27.05 (C2), 26.68 (C15), 26.37 (C16), 23.15 (C27), 18.65 (C26), 17.46 (C6), 16.36 (C25), 15.57 (C24); HRMS (m/z): $[\mathrm{M}+\mathrm{H}]^{+}$calcd $\mathrm{C}_{37} \mathrm{H}_{59} \mathrm{~N}_{2} \mathrm{O}_{5}$ : 611.44240, found: 611.44608 .

General procedure for preparation of compound (3) and bisamide (5). Piperazine (0.35 g, $4.0 \mathrm{mmol})$ was dissolved in acetonitrile $(20 \mathrm{~mL})$, dropped with acetonitrile solution of the intermediate (6) (0.59 g, $1.0 \mathrm{mmol}$ ), and the mixture was stirred under reflux for $12 \mathrm{~h}$. The solvent was removed under vacuum to give a residue. The residue was treated with a mixture of ethanol and water. The solution was stirred at room temperature for $30 \mathrm{~min}$, and a solid was obtained by filtration while washing with $\mathrm{H}_{2} \mathrm{O}$. Finally, the mixture was purified by silica gel column to afford the bisamide (5) and the desired product (3), and the isolated yields were $53.7 \%$ and $40.8 \%$, respectively.

Bisamide (5) a white solid; yield, $53.7 \%$; $\mathrm{mp} 211.4-212.0^{\circ} \mathrm{C}$. HRMS $(m / z):[\mathrm{M}+\mathrm{Na}]^{+}$calcd for $\mathrm{C}_{64} \mathrm{H}_{98} \mathrm{~N}_{2} \mathrm{NaO}_{6}: 1013.73226$, found: 1013.73499 .

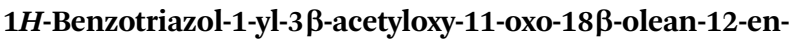
30-oate (6). A white solid; yield, 97.2\%; mp 263.4-264.4 ${ }^{\circ} \mathrm{C} .{ }^{7}$

Compound (7). Compound $3(0.54 \mathrm{~g}, 1.0 \mathrm{mmol})$ and triethylamine ( $0.13 \mathrm{~g}, 1.2 \mathrm{mmol})$ were dissolved in $\mathrm{CH}_{2} \mathrm{Cl}_{2}(20 \mathrm{~mL})$ at $0{ }^{\circ} \mathrm{C}$ under stirring. Substituted acyl chloride $(1.0 \mathrm{mmol})$ was added, and the reaction was stirred at $40{ }^{\circ} \mathrm{C}$. After reaction, the mixture was washed with saturated aqueous $\mathrm{NaHCO}_{3}$ and water. The organic layer was dried over anhydrous $\mathrm{Na}_{2} \mathrm{SO}_{4}$, filtered, and concentrated under reduced pressure. The crude product was then chromatographed on silica $\left(20: 1 \mathrm{CH}_{2} \mathrm{Cl}_{2}-\right.$ methanol).

A white solid; yield, $38.4 \%$; mp 219.6-220.4 ${ }^{\circ} \mathrm{C}$. $\operatorname{HRMS}(\mathrm{m} / \mathrm{z})$ : $[\mathrm{M}+\mathrm{Na}]^{+}$calcd for $\mathrm{C}_{50} \mathrm{H}_{64} \mathrm{Cl}_{2} \mathrm{~N}_{2} \mathrm{NaO}_{5}:$ 865.40900, found: 865.41315.

\subsection{Primary anticancer assay}

All the cell lines used in this study were either purchased from Shanghai Institutes for Biological Sciences, Chinese Academy of Sciences (A549, HepG2, MCF-7, PC-3 and LO2) or from Nanjing Cobioer Biosciences Co., Ltd. (Karpas299). Cells were incubated with $5 \% \mathrm{CO}_{2}$ at $37{ }^{\circ} \mathrm{C}$ for $24 \mathrm{~h}$. Subsequently the compounds and the reference were dissolved into the culture medium. Then, the cells were treated with different concentrations of test compounds and further incubated. After drug treatment, $20 \mu \mathrm{L}$ of MTT solution at $5 \mathrm{mg} \mathrm{mL}^{-1}$ was added and incubated for $4 \mathrm{~h}$. $100 \mu \mathrm{L}$ of DMSO was added into each well to dissolve the purple formazan formed. The absorbance was determined at $630 \mathrm{~nm}$ using a plate reader. The $\mathrm{IC}_{50}$ was calculated using GraphPad Prism version 6.0 software (San Diego, USA) from the non-linear curve.

\subsection{Kinase activity determination}

The effects of the compounds on the activities of the tyrosine kinase was determined using enzyme-linked immunosorbent assay (ELISA). Briefly, various concentrations of test compounds diluted in DMSO were then added to each well. DMSO was used as the negative control. The kinase reaction initiated at $37^{\circ} \mathrm{C}$ for 60 min after the addition of biotinylated detection Ab working solution. After being washed, HRP affinipure goat anti-mouse IgG $(\mathrm{H}+\mathrm{L})$ was added. The plate was then incubated at $37{ }^{\circ} \mathrm{C}$ for $30 \mathrm{~min}$. After being washed, the substrate reagent was added to each well at $37{ }^{\circ} \mathrm{C}$. The enzyme-substrate reaction is terminated by the addition of stop solution as the color changed, and the plate was analyzed using a multi-well spectrophotometer at $450 \mathrm{~nm}$. The $\mathrm{IC}_{50}$ values were calculated from the inhibition curves in two separate experiments.

\subsection{Molecular modeling}

The prepared compounds were drawn using the ChemBioDraw Ultra 14.0 software, and then were saved in the sdf format, these molecules were imported into the Discovery Studio 3.5 software for the docking study (the Discovery Studio 3.5 software package, Accelrys, Co. Ltd., San Diego, USA). And those better docking conformations obtained from the full minimization protocol. Molecular docking into the 3D X-ray structure of ALK kinase (PDB code: 2XP2) was performed by using CDOCKER protocol. All the water and ligand were removed from the protein and the polar hydrogen was added. Molecular docking was validated by the docking of the co-crystallized inhibitor for enzyme, and root-mean-square deviation (RMSD) value for the backbone atoms between docked pose and crystallographic pose was below $1.5 \AA$.

The molecular overlay program of the Discovery Studio 3.5 software was used to align moleculars and calculate molecular similarity according to the default parameter $(50 \%$ steric field and $50 \%$ electrostatic field).

\section{Conflicts of interest}

There are no conflicts to declare.

\section{Acknowledgements}

This work was supported by the Natural Science Foundation of Liaoning Province (No. 2019-ZD-0693 and No. 20170540396), General Research Projects of Liaoning Provincial Department of 
Education (No. JQL201715410) and Undergraduate Innovation and Entrepreneurship Training Program (No. 2019052).

\section{References}

1 Y. Liu and N. S. Gray, Nat. Chem. Biol., 2006, 2, 358-364.

2 A. Backes, B. Zech, B. Felber, B. Klebl and G. Müller, Expert Opin. Drug Discovery, 2008, 3, 1427-1449.

3 Y. Niu, X. Yao and H. Ji, RSC Adv., 2019, 9, 12441-12454.

4 A. Roohbakhsh, M. Iranshahy and M. Iranshahi, Curr. Med. Chem., 2016, 23, 498-517.

5 H. Hussain, I. R. Green, U. Shamraiz, M. Saleem, A. Badshah, G. Abbas, N. U. Rehman and M. Irshad, Expert Opin. Ther. Pat., 2018, 28, 383-398.

6 X. Li, Y. Liu, N. Wang, Y. Liu, S. Wang, H. Wang, A. Li and S. Ren, RSC Adv., 2019, 9, 27294-27304.

7 D. Cai, Z. Zhang, Y. Chen, Y. Zhang, Y. Sun and Y. Gong, Molecules, 2019, 24, 3631.

8 D. P. Alho, J. A. Salvador, M. Cascante and S. Marin, Molecules, 2019, 24, 766.

9 B. Xu, G.-R. Wu, X.-Y. Zhang, M.-M. Yan, R. Zhao, N.-N. Xue, K. Fang, H. Wang, M. Chen and W.-B. Guo, Molecules, 2017, 22, 924.

10 T. L. Yan, L. F. Bai, H. L. Zhu, W. M. Zhang and P. C. Lv, ChemMedChem, 2017, 12, 1087-1096.

11 R. Sharma, S. K. Guru, S. K. Jain, A. S. Pathania, R. A. Vishwakarma, S. Bhushan and S. B. Bharate, MedChemComm, 2015, 6, 564-575.
12 R. Csuk, S. Schwarz, R. Kluge and D. Ströhl, Eur. J. Med. Chem., 2010, 45, 5718-5723.

13 J. Liu and S. Ma, Curr. Med. Chem., 2017, 24, 590-613.

14 X. Yan, C. Liao, Z. Liu, A. T. Hagler, Q. Gu and J. Xu, Curr. Drug Targets, 2016, 17, 1580-1585.

15 H. Eckert and J. Bajorath, Drug Discovery Today, 2007, 12, 225-233.

16 F. Barbosa and D. Horvath, Curr. Top. Med. Chem., 2004, 4, 589-600.

17 P. J. Ballester and W. G. Richards, Proc. R. Soc. A, 2007, 463, 1307-1321.

18 C. Ferroud, M. Godart, S. Ung, H. Borderies and A. Guy, Tetrahedron Lett., 2008, 49, 3004-3008.

19 W.-C. Chou, M.-C. Chou, Y.-Y. Lu and S.-F. Chen, Tetrahedron Lett., 1999, 40, 3419-3422.

20 M. H. Abdelrahman, B. G. Youssif, A. H. Abdelazeem, H. M. Ibrahim, A. M. Abd El Ghany, L. Treamblu and S. N. A. Bukhari, Eur. J. Med. Chem., 2017, 127, 972-985.

21 B. Lallemand, F. Chaix, M. Bury, C. Bruyère, J. Ghostin, J.-P. Becker, C. Delporte, M. Gelbcke, V. Mathieu, J. Dubois, M. Prévost, I. Jabin and R. Kiss, J. Med. Chem., 2011, 54, 6501-6513.

22 J. J. Cui, M. Tran-Dubé, H. Shen, M. Nambu, P.-P. Kung, M. Pairish, L. Jia, J. Meng, L. Funk and I. Botrous, J. Med. Chem., 2011, 54, 6342-6363.

23 S. Sommerwerk, L. Heller, C. Kerzig, A. E. Kramell and R. Csuk, Eur. J. Med. Chem., 2017, 127, 1-9. 